\title{
SPECTROSCOPIC OBSERVATIONS OF PLANETARY NEBULAE IN THE NORTHERN SPUR OF M31
}

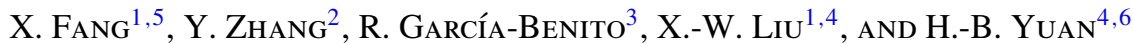 \\ ${ }^{1}$ Department of Astronomy, School of Physics, Peking University, Beijing 100871, China; fangx@pku.edu.cn \\ 2 Department of Physics, University of Hong Kong, Pokfulam Road, Hong Kong, China \\ ${ }^{3}$ Instituto de Astrofísica de Andalucía (CSIC), Glorieta de la Astronomía s/n, E-18008 Granada, Spain \\ ${ }^{4}$ Kavli Institute for Astronomy and Astrophysics, Peking University, Beijing 100871, China \\ Received 2013 May 27; accepted 2013 July 16; published 2013 August 26
}

\begin{abstract}
We present spectroscopy of three planetary nebulae (PNe) in the Northern Spur of the Andromeda galaxy (M31) obtained with the Double Spectrograph on the $5.1 \mathrm{~m}$ Hale Telescope at the Palomar Observatory. The samples were selected from the observations of Merrett et al. Our purpose is to investigate the formation of the substructures of M31 using PNe as a tracer of chemical abundances. The [O III] $\lambda 4363$ line is detected in the spectra of two objects, enabling temperature determinations. Ionic abundances are derived from the observed collisionally excited lines, and elemental abundances of nitrogen, oxygen, neon, sulfur, and argon are estimated. We study the correlations between oxygen and the $\alpha$-element abundance ratios using our sample and the M31 disk and bulge PNe from the literature. In one of the three $\mathrm{PNe}$, we observed a relatively higher oxygen abundance compared to the disk sample of M31 at similar galactocentric distances. The results of at least one of the three Northern Spur PNe might be in line with the proposed possible origin of the Northern Spur substructure of M31, i.e., the Northern Spur is connected to the Southern Stream and both substructures comprise the tidal debris of the satellite galaxies of M31.
\end{abstract}

Key words: galaxies: abundances - galaxies: formation - galaxies: individual (M31) - ISM:

abundances - planetary nebulae: general - stars: evolution

Online-only material: color figures

\section{INTRODUCTION}

In hierarchical cosmology, accretion (or merging) of smaller galaxies contributes significantly to the growth of large galaxies (e.g., the classical theoretical work by White 1978; White \& Rees 1978), especially in the early evolutionary stages of the larger structures. The most recent observational studies of the growth of galaxies were carried out by the CALIFA ${ }^{7}$ group (Pérez et al. 2013), who analyzed 105 galaxies in the largest three-dimensional spectroscopic survey of galaxies in the local universe. The outskirts of galaxies hold fundamental clues about the formation histories of galaxies. It is into these regions that new material continues to arrive as part of ongoing assembly processes. Furthermore, these locations mark the deposition sites during violent interactions in a galaxy's distant past. Since it takes a very long time for the accreted material to be erased by the process of phase mixing (due to the long dynamical timescales), we can detect various substructures, e.g., tidal tails, that are expected to exist in the areas beyond the disk of a large galaxy. The tidal disruption of these small systems is expected to result in loosely bounded stars surrounding the galaxy, at distances up to 10-100 times the radius of the central disk (Abadi et al. 2006). Substructures like stellar streams have been observed in both the Milky Way (MW) and other large spiral galaxies. The Sagittarius Dwarf Galaxy provides a fine example of stellar streams near the MW (e.g., Ibata et al. 2001b; Majewski et al. 2003).

The Andromeda galaxy (M31) is the nearest large spiral system and one of the best candidates for studying the

\footnotetext{
5 Current address: Kavli Institute for Astronomy and Astrophysics, Peking University, Beijing 100871, China.

6 LAMOST Fellow.

7 Calar Alto Legacy Integral Field spectroscopy Area Survey. URL: http://califa.caha.es.
}

debris of interaction. A large number of coherent stellar substructures have been detected in its halo and outer disk (e.g., Ibata et al. 2001a, 2007; Ferguson et al. 2002; Irwin et al. 2005; McConnachie et al. 2003, 2004, 2009). The number, luminosity, morphology, and stellar populations of the relics provide important clues to the assemblage history of M31. However, a comprehensive survey of those relics is quite difficult given their intrinsic faintness, dense distribution, and the vast space over which the stars are spread. Those factors make the stars and clusters in M31 not ideal tools for abundance studies. Besides, there is also contamination from the foreground stars of the MW. Planetary nebulae (PNe) are excellent tracers for studying the chemistry, kinematics, and stellar contents of the substructures because they are bright but not packed too closely. PNe are easily detectable at the distance of M31 $(785 \mathrm{kpc}$; McConnachie et al. 2005) given their bright, narrow emission-line spectra. They are also one of the best candidates that can provide both very accurate velocities and precision abundance measurements of elements such as $\mathrm{O}, \mathrm{He}, \mathrm{Ne}, \mathrm{N}, \mathrm{Ar}$, and $\mathrm{S}$. Observations and chemical studies of $\mathrm{PNe}$ (as well as $\mathrm{H}$ II regions) in M31 were carried out decades ago (e.g., Ford \& Jacoby 1978a, 1978b; Jacoby \& Ford 1986; Stasińska et al. 1998; Richer et al. 1999; Jacoby \& Ciardullo 1999; the most recent observations are by Kwitter et al. 2012, Zurita \& Bresolin 2012, and Sanders et al. 2012). Using the nebular sample available, oxygen abundance gradients in M31 have been derived that further our understanding of the chemical evolution of the galaxy.

A number of substructures have been observed in the outer halo of M31. The Northern Spur is a peculiar low surface brightness structure extending out of M31's disk, that contains a metal-rich stellar population. Decades ago, it was observed to lie in the direction of M31's gaseous warp (e.g., Newton \& Emerson 1977). Innanen et al. (1982) noticed the antisymmetrical warping of the stellar disk along the major axis of M31 through digital stacking of Palomar Schmidt plates 
Table 1

Properties and Observations of the Three Northern Spur PNe ${ }^{\mathrm{a}}$

\begin{tabular}{|c|c|c|c|c|c|c|c|}
\hline \multirow[t]{2}{*}{$\mathrm{PN} \mathrm{ID}^{\mathrm{b}}$} & \multirow{2}{*}{$\begin{array}{c}\text { R.A. } \\
(\mathrm{J} 2000.0)\end{array}$} & \multirow{2}{*}{$\begin{array}{c}\text { Decl. } \\
(\mathrm{J} 2000.0)\end{array}$} & \multirow[t]{2}{*}{$m(\lambda 5007)^{\mathrm{c}}$} & \multirow{2}{*}{$\begin{array}{c}v_{\text {helio }} \\
\left(\mathrm{km} \mathrm{s}^{-1}\right)\end{array}$} & \multirow{2}{*}{$\begin{array}{l}R_{\mathrm{gal}}{ }^{\mathrm{d}} \\
(\mathrm{kpc})\end{array}$} & \multicolumn{2}{|c|}{ DBSP Exp. (s) } \\
\hline & & & & & & Blue Arm & Red Arm \\
\hline PN1 (2426) & $0: 47: 00.69$ & $42: 58: 55.20$ & 20.61 & -19.6 & 25.9 & $8 \times 1800$ & $8 \times 1800$ \\
\hline PN2 (2431) & $0: 47: 58.60$ & 43:00:06.48 & 20.83 & -44.4 & 27.2 & $8 \times 1800$ & $8 \times 1800$ \\
\hline PN3 (2421) & $0: 45: 42.60$ & $42: 55: 26.30$ & 20.84 & -90.3 & 23.9 & $6 \times 1800$ & $6 \times 1800$ \\
\hline
\end{tabular}

Notes.

a Observations were carried out with a slit width of 1.5 arcsec.

${ }^{b}$ Number in the bracket that follows the PN ID is the ID number from Merrett et al. (2006).

${ }^{c} m(\lambda 5007)=-2.5 \log F(\lambda 5007)-13.74$.

${ }^{\mathrm{d}}$ Sky-projected galactocentric distance given by Merrett et al. (2006).

of the galaxy. Walterbos \& Kennicutt (1988) observed a faint light bending away from the northern major axis using multicolor photometry and attributed that effect to either a possible galactic reflection nebula or a warp in the outer stellar disk of M31. However, the results for the northern outer disk of M31, according to Walterbos \& Kennicutt (1988), were not conclusive due to the faintness of the emission there. The warp observed by both Innanen et al. (1982) and Walterbos \& Kennicutt (1988) to the northeastern major axis of M31 starts at about $18 \mathrm{kpc}$ from the galactic center and extends to the region well beyond $\sim 25 \mathrm{kpc}$. Since the Northern Spur lies in the direction of M31's gaseous warp, its projection away from the galactic plane was usually attributed to a severe warp in the stellar disk.

Progress in observational and data-processing techniques during the past decade has enabled large surveys. The faint substructures of M31 can now be studied in greater detail; consequently, the properties of these substructures can be better understood. Ibata et al. (2001a) discovered a giant stream (i.e., the Southern Stream) of metal-rich stars within the halo of M31 and attributed the possible source of the stream to the dwarf galaxies M32 and NGC 205, which are close companions of M31 and might have lost a substantial number of stars due to tidal interactions. Ibata et al. (2001a) concluded that the epoch of galaxy building of M31 still continues and that tidal streams might be a generic feature of galaxy halos. Ferguson et al. (2002) carried out a panoramic survey of the halo and outer disk of M31 and studied the density and color distribution of red giant branch (RGB) stars. These authors confirmed the Southern Stream first announced by Ibata et al. (2001a) and found an enhancement in both stellar density and metallicity in the Northern Spur. Inspired by the asymmetry of the Southern Stream (i.e., it does not appear to the northwest), Ferguson et al. (2002) hypothesized that the Southern Stream might be associated with the Northern Spur. McConnachie et al. (2003) measured a radial distance change along the Stream by analyzing the metal-rich RGB luminosity function and derived an angle of $60^{\circ}$ of the stream to the line of sight. Combining the distance gradient with the angular extent, McConnachie et al. (2003) found that the Southern Stream extends from approximately $100 \mathrm{kpc}$ behind to $40 \mathrm{kpc}$ in front of M31. Merrett et al. (2003) proposed that Southern Stream might be connected to the Northern Spur and presented a threedimensional orbit for that connection by studying the dynamics of PNe in the disk of M31. They also suggested that M32 might be the source of the two substructures mainly because the satellite is found to coincide with the Southern Stream in both spatial position and velocity.

Currently, the exact origin of the Northern Spur is still largely unknown, and the dynamical model proposed by Merrett et al. (2003) needs to be tested. However, observational data in this region are scarce. In order to assess the hypothesis of the origin of the Northern Spur by studying its chemistry, we present spectroscopic observations of PNe in this substructure and deduce their elemental abundances from emission lines detected in the spectra. This investigation is the first chemical study of the PNe in the Northern Spur with spectroscopy. Section 2 presents the observations and data reduction and discusses the observing challenges we encountered in studying the PNe in the Northern Spur. Line flux measurements, plasma diagnostics, and abundance determinations are given in Section 3. A comparison of the abundances in our sample with those in the M31 disk and bulge PNe (also H II regions) is presented in Section 4. A discussion of the possible origin of the Northern Spur also appears in Section 4. A summary and conclusions are given in Section 5.

\section{OBSERVATIONS AND DATA REDUCTION}

\subsection{Target Selection}

The main purpose of the current observations is to understand the formation history of substructure in the outer disk of M31 by studying the chemistry of PNe in the Northern Spur. In order to obtain accurate measurements of the heavy element abundances, we need to know the physical conditions, most importantly, the electron temperatures of the PNe. However, the heliocentric velocity of M31 $\left(\sim-300 \mathrm{~km} \mathrm{~s}^{-1}\right)$, combined with the very bright mercury line $\mathrm{Hg} \mathrm{I} \lambda 4358.34^{8}$ from the nearby urban area, makes the [O III] $\lambda 4363.21$ auroral line difficult to resolve. In order to increase the likelihood that the [O III] $\lambda 4363$ line can be measured, PNe with the lowest radial velocities are favored. Candidates for observations were selected from Merrett et al. (2006). Most of the candidates have either low radial velocities but also low surface brightnesses (i.e., with the magnitude of the [O III] $\lambda 5007$ line $m_{5007}>21.5$ ) or relatively high surface brightnesses but also high radial velocities. Finally, a compromise between the magnitude of the [O III] $\lambda 5007$ line and radial velocity, as well as the consideration of time, resulted in three objects for observation. Table 1 presents the coordinates (right ascension and declination, hereafter R.A. and decl., respectively), apparent magnitudes at [O III] $\lambda 5007$, heliocentric velocities, and galactocentric distances of the three $\mathrm{PNe}$ (hereafter named PN1, PN2, and PN3). These PNe are located in the outer disk of M31 and have been identified as being in the Northern Spur substructure by Merrett et al. (2006).

\footnotetext{
8 The wavelength of this line is adopted from the National Institute of Standards and Technology (NIST) Atomic Spectra Database. URL: http://www.nist.gov/pml/data/asd.cfm. The data source is Sansonetti et al. (1996).
} 


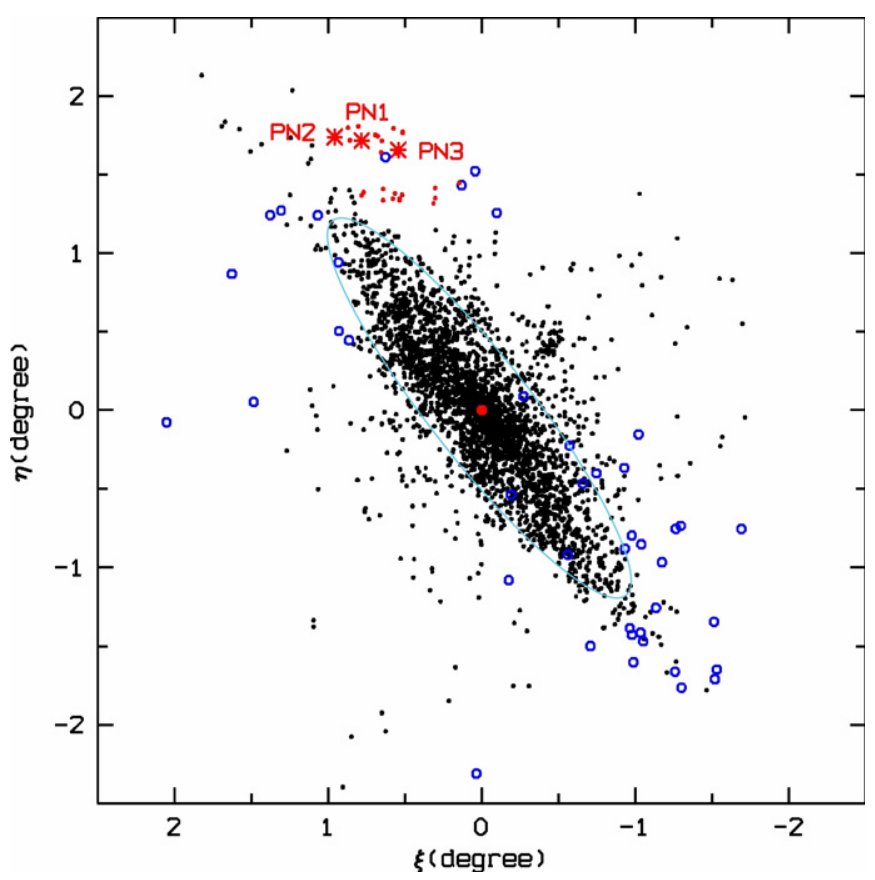

Figure 1. M31 PNe observed by Merrett et al. (2006; black dots) and LAMOST (Yuan et al. 2010; blue open circles). The small red dots to the north of the M31 disk are the PNe in the Northern Spur identified by Merrett et al. (2006). The three Northern Spur PNe observed and studied in the current paper, namely, PN1, PN2, and PN3, are indicated by the red asterisks. The center of M31 is indicated with a red dot. The coordinates $\xi$ and $\eta$ represent the M31-based reference frame defined by Huchra et al. (1991). The green ellipse is a disk with a $2^{\circ}$ radius $(27.4 \mathrm{kpc})$ around the center of $\mathrm{M} 31$, assuming an inclination angle of 77.7 and a position angle of 37.7 (de Vaucouleurs 1958) for the M31 disk.

(A color version of this figure is available in the online journal.)

Their projected galactocentric distances range from 23 to $27 \mathrm{kpc}$. Figure 1 shows the spatial distribution of PNe observed in M31. The sample includes $~ 3000$ PNe from Merrett et al. (2006) and those observed by LAMOST $^{9}$ (Yuan et al. 2010). The coordinates $\xi$ and $\eta$ in Figure 1 are the offsets in R.A. and decl. relative to the center of M31, respectively, and thus define an M31-based reference frame. $\xi$ and $\eta$ are calculated following the geometric transformations of Huchra et al. (1991):

$$
\xi=\sin (\text { R.A. }- \text { R.A. } 0) \cos (\text { decl. })
$$

and

$$
\begin{aligned}
\eta= & \sin (\text { decl. }) \cos \left(\text { decl. }_{0}\right) \\
& -\cos (\text { R.A. }- \text { R.A. } .0) \cos (\text { decl. }) \sin \left(\text { decl. }_{0}\right)
\end{aligned}
$$

where R.A. $0=00^{\mathrm{h}} 42^{\mathrm{m}} 44.4(\mathrm{~J} 2000.0)$ and decl. $0=41^{\circ} 16^{\prime} 08^{\prime \prime}$ (J2000.0) are the coordinates of the optical center of M31 and are adopted from de Vaucouleurs et al. (1991). The radial velocities of PN1, PN2, and PN3 are $-19.6,-44.4$, and $-90.3 \mathrm{~km} \mathrm{~s}^{-1}$, respectively. Thus, the corresponding wavelength differences between the observed [O III] $\lambda 4363$ auroral line and the mercury line at $\lambda 4358$ for the three objects are $4.59,4.23$, and $3.56 \AA$. Given that the width of the mercury line is 5-6 $\AA$ and the FWHM of a nebular emission line is $\sim 2.40 \AA$ for the blue spectra, the [O III] $\lambda 4363$ auroral line is expected to be resolved from the mercury line for all three objects, provided that the subtraction of the sky background is good.

\footnotetext{
9 Large Sky Area Multi-Object Fiber Spectroscopic Telescope. URL: http://www.lamost.org.
}

\subsection{Observations}

We observed three PNe in the Northern Spur outer disk of M31 with the Double Spectrograph (DBSP) on the Palomar $5.1 \mathrm{~m}$ Hale Telescope. The dichroic D48, which splits light into separate blue and red channels around $4800 \AA$, was used. The two channels cover the wavelength ranges 3400-4900 $\AA$ and 4800-7300 $\AA$. In the observations, the DBSP configuration consists of a 1200 line $\mathrm{mm}^{-1}$ grating blazed at $4150 \AA$ (with a grating angle of 34.75) in the blue arm and a 316 line $\mathrm{mm}^{-1}$ grating blazed at $6050 \AA$ (with a grating angle of 23.25) in the red channel. The blue channel has a thinned, anti-reflectivecoated $2048 \times 4096$ (in the 4096 dispersion axis) CCD with $15 \mu \mathrm{m}$ pixels, and the red channel has a thinned $4096 \times$ 2048 CCD with $15 \mu \mathrm{m}$ pixels. Given the seeing conditions $(\sim 1.5 \mathrm{arcsec})$ at Palomar, the slit width was set to be $1.5 \mathrm{arcsec}$; the slit length is 128 arcsec. This setup enabled us to perform spectroscopy over the wavelength range 3400-4900 $\AA$ with a resolution of $2.4 \AA$ (FWHM) at $0.55 \AA$ pixel $^{-1}$, and over the wavelength range $4800-7300 \AA$ with a resolution of $6.9 \AA$ (FWHM) at $2.45 \AA$ pixel ${ }^{-1}$.

Observations were taken on two nights (Table 1). On UT date 2011 September 22, we observed two PNe (PN1 and PN2). The observations consisted of eight $1800 \mathrm{~s}$ exposures of PN1 and four $1800 \mathrm{~s}$ exposures of PN2 (for both the blue and red channels of the DBSP). The observations were taken under photometric conditions with a typical seeing of 1.5 arcsec. On September 23, we observed PN2 and PN3. The observations consisted of four 1800 s exposures of PN2 and six 1800 s exposures of PN3. The limiting magnitude of the guide star CCD on the telescope was approximately $16 \mathrm{mag}$, while our target PNe were much fainter. In order to locate the faint targets in the slit, we adopted the so-called blind offset technique: first, we placed the slit on a selected bright guide star near each target PN and then moved the slit to the target position. In order to avoid light losses due to atmospheric diffraction, the slit was placed along the parallactic angle during the observations. The parallactic angle was calculated by the telescope control system. The slit was rotated every 30-60 minutes during the observations. The data from both nights were calibrated via a series of exposures of the spectrophotometric standard stars LDS749B and $\mathrm{Hz} 2$, which were selected from the ESO Standard Stars Catalogs. ${ }^{10}$ Arc lines of an FeAr lamp were used for wavelength calibration for the blue spectra of the three PNe, and an HeArNe lamp was used for the red spectra. The lamp exposures were obtained before the observations of each PN target so that the errors in the wavelength calibration were minimized.

\subsection{Data Reduction}

All of the data were reduced with standard procedures for long-slit spectra using the LONG92 package in MIDAs. ${ }^{11}$ The raw two-dimensional (2D) spectra were debiased, flat-fielded, and cosmic-ray removed, and then wavelength calibrated using exposures of an FeAr (for the blue data) and an HeArNe (for the red data) lamp. The sky background was then subtracted from the 2D spectra. One-dimensional (1D) spectra of the PNe were extracted from the 2D spectra, corrected for atmospheric extinction, and flux calibrated using observations of the standard

\footnotetext{
10 URL: http://www.eso.org/sci/observing/tools/standards/spectral.html. The ultraviolet and optical spectrophotometric data of LDS749B are given by Oke (1990), and those of $\mathrm{Hz} 2$ are from the unpublished data of J. B. Oke.

11 MIDAS is developed and distributed by the European Southern Observatory.
} 


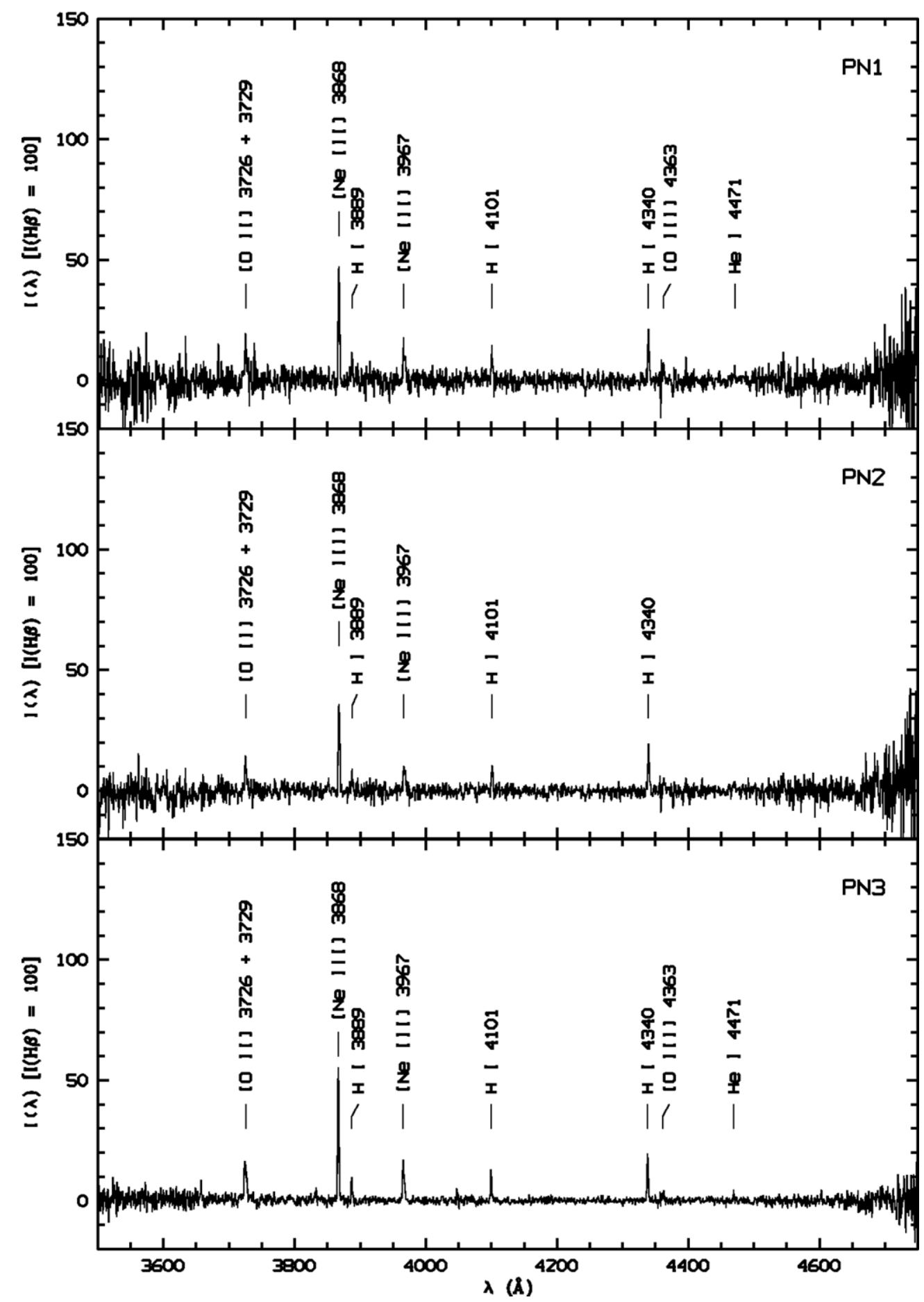

Figure 2. DBSP blue spectra of PN1 (upper panel), PN2 (middle panel), and PN3 (lower panel), with important emission lines labeled. All spectra have been normalized such that $\mathrm{H} \beta$ has an integrated flux of 100 and extinction has been corrected for.

stars LDS749B and $\mathrm{Hz} 2$. The 1D spectra with $1800 \mathrm{~s}$ exposures for each PN were combined, ${ }^{12}$ corrected for interstellar extinction (see Section 2.4), and then normalized such that $\mathrm{H} \beta$ had an integrated flux of 100 . Here, the $\mathrm{H} \gamma$ line was used for the blue spectra and $\mathrm{H} \alpha$ was used for the red spectra, assuming that the $I(\mathrm{H} \gamma) / I(\mathrm{H} \beta)$ and $I(\mathrm{H} \alpha) / I(\mathrm{H} \beta)$ ratios of $\mathrm{H}$ I are those calculated by Storey \& Hummer (1995) at $T_{\mathrm{e}}=10,000 \mathrm{~K}$ and

\footnotetext{
12 The spectra were combined using a weighted average of individual exposures, with assigned weights proportional to the $\mathrm{S} / \mathrm{N}$ of the same emission line detected in the blue or red spectrum.
}

$N_{\mathrm{e}}=10^{4} \mathrm{~cm}^{-3}$ for Case B recombination. Figures 2 and 3 are the blue and red 1D spectra of the three PNe, respectively. The signal-to-noise ratios (S/Ns) of the blue spectra are systematically lower than those of the red data; this effect is mainly due to the relatively low efficiency of the blue CCD.

Observations of the three PNe in the Northern Spur of M31 are challenging, given that the sky background at the Palomar Observatory is strong and our targets are faint. The very bright mercury line at $4358 \AA$ makes it difficult to directly observe the [O III] $\lambda 4363$ auroral line (Section 2.1), where the latter can be used to derive the electron temperature. We managed to detect 


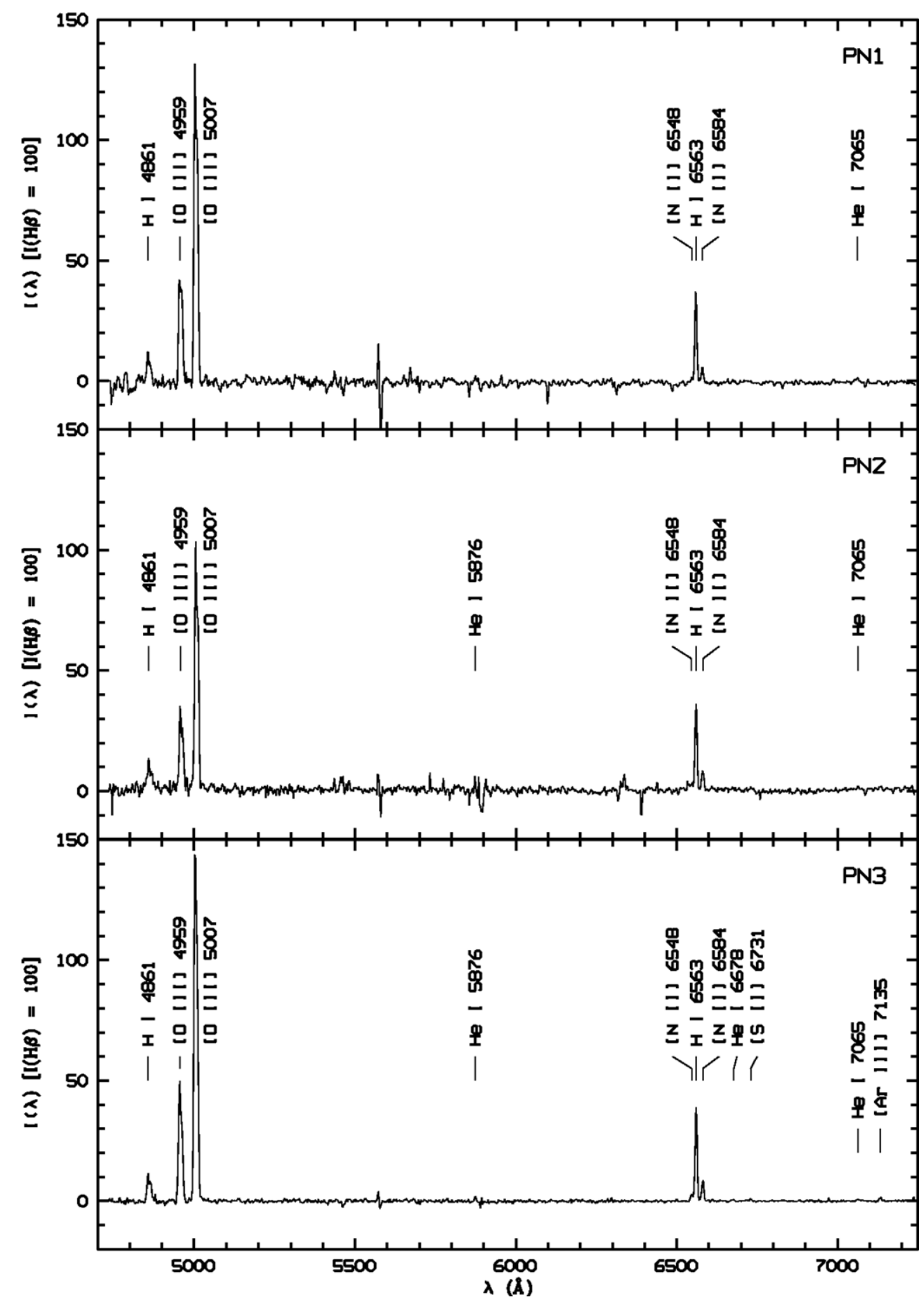

Figure 3. Same as Figure 2 but for the DBSP red spectra.

emission lines in the three PNe and separated the [O III] $\lambda 4363$ line from the mercury line in two objects. Figures 4-6 show the 2D blue spectra of PN1, PN2, and PN3, respectively, over the wavelength region 3710-4405 $\AA$. The [O III] $\lambda 4363$ auroral line can be seen in the sky-subtracted spectra of PN1 and PN3. However, residuals from sky subtraction, especially of the strong mercury line, are still present in the processed 2D image (see the lower panels of Figures 4-6) because sky subtraction was done by choosing two separate sections on the slit, one on either side of the target spectrum, with a separation of about 3-5 pixels from the PN. This technique minimizes the effects of image distortion along the slit.
Consideration has been given to the distortion of emission lines. We tried to map out the distortion of the $\mathrm{Hg}$ I $\lambda 4358$ mercury line along the slit by measuring the peak wavelengths of the mercury line in the wavelength-calibrated 2D image along the slit direction, with every five rows of CCD pixels binned together. The peak positions of the mercury line were well fit with a linear regression. The linear fit yields a very small angle relative to the slit direction, which indicates that the distortion is small. We also checked the distribution of the mercury line flux along the slit and found that it is mostly homogeneous. Besides, the sky background regions defined on the slit (two regions on the slit, with one on either side of a target $\mathrm{PN}$ ) are very close to 

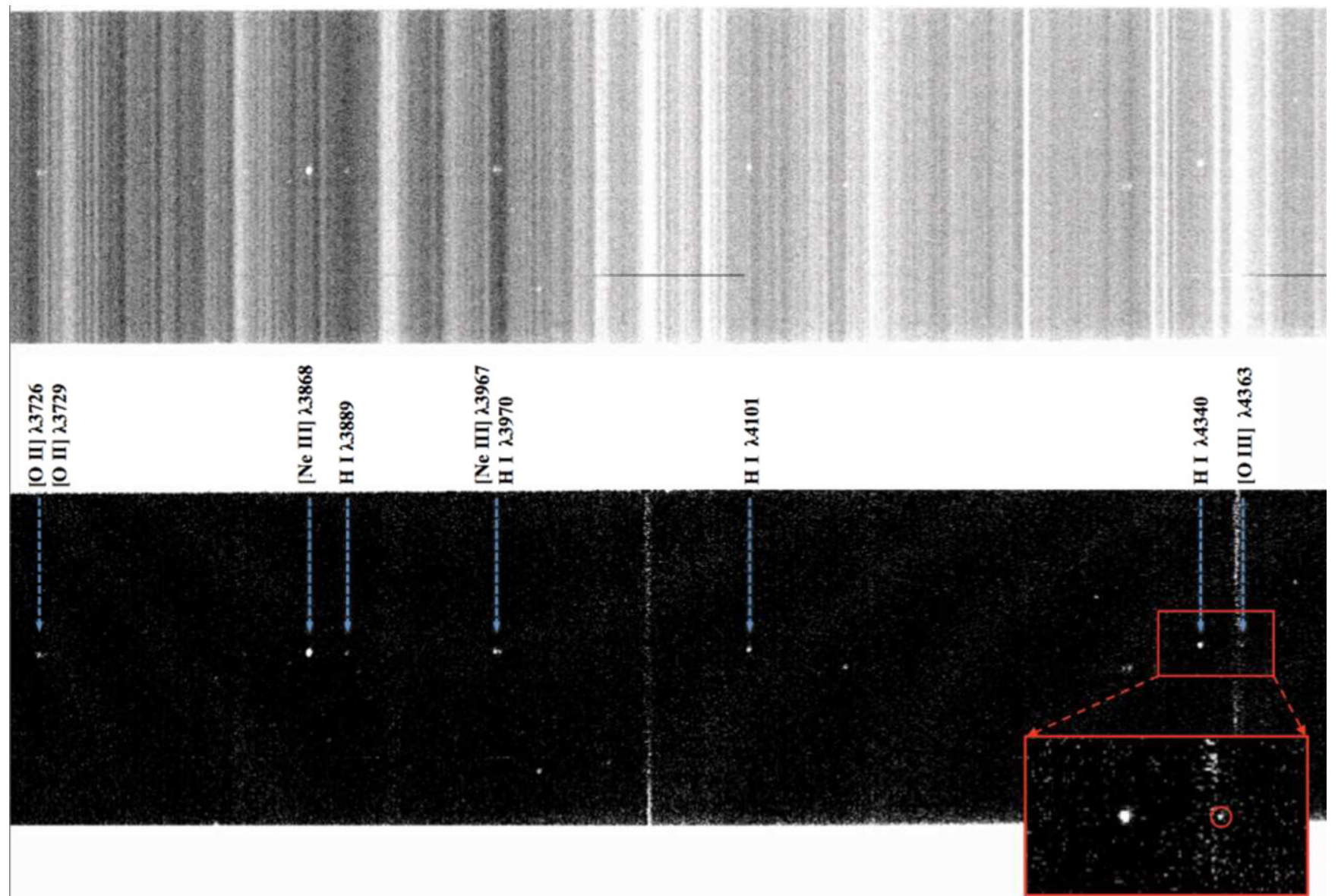

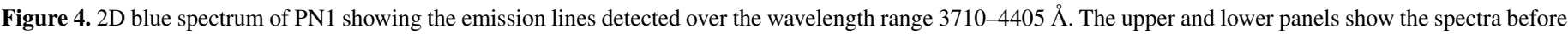

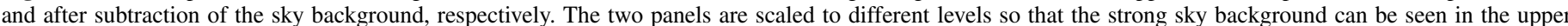

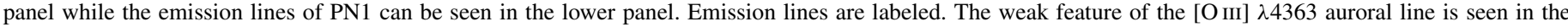

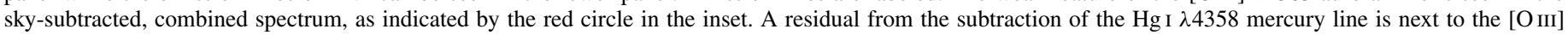
$\lambda 4363$ line.

(A color version of this figure is available in the online journal.)

the target PN. Thus, the effects of distortion of the sky lines on the PN emission line measurements are negligible. Nevertheless, we applied the distortion correction to all 2D frames. Despite much effort, not much can be further improved in regards to the sky subtraction. Given the relatively strong sky background compared to the intrinsic faintness of our target PNe, it is difficult to completely remove the sky background over the whole slit in the 2D frame.

Since the PNe in M31 are point sources, emission lines on a $2 \mathrm{D}$ spectrum are detected as bright spots (see Figures 4-6) with diameters of 5-10 pixels depending on the strength of the line. The dispersion direction of the blue spectrum is not always perpendicular to the slit. We carried out polynomial fits to the positions (wavelength in $\AA$ versus the row number of the CCD along the slit) of emission lines on the wavelength-calibrated and sky-subtracted 2D frame, and then extracted the 1D spectrum by averaging a number of pixels in the direction of the slit (about 20 pixels with the target $\mathrm{PN}$ in the center) along the track of the polynomial fit. Although residuals from sky subtraction are present in the 2D image, they are only the strongest far away from the target PN and negligible in the region close by, as seen in Figures 4 and 6. Thus, measurements of the [O III] $\lambda 4363$ line are not affected much. The dispersion direction of the red spectra is perpendicular to the slit, and we averaged the CCD rows along the slit directly to obtain the $1 \mathrm{D}$ spectra. The spectra of the two standards (LDS749B and $\mathrm{Hz} 2$ ) were used to derive the response curves of the blue and red channels for the dichroic D48. The efficiency is very low above $4700 \AA$ in the blue channel and below $4800 \AA$ in the red channel. The extinction-corrected 1D blue spectra are low quality above $4700 \AA$, as seen in Figure 2; and the $\mathrm{H} \beta$ line cannot be used to join the blue and red spectra, as expected. Given the relatively low efficiency near $4800 \AA$ on the red $\mathrm{CCD}$, we used the $\mathrm{H} \alpha$ line instead of $\mathrm{H} \beta$ to normalize the line fluxes such that $I(\mathrm{H} \alpha)=285$. For the blue spectra, all line fluxes were scaled such that $I(\mathrm{H} \gamma)=47$. The normalization is based on the theoretical $\mathrm{H}$ I line ratios and the assumption that $I(\mathrm{H} \beta)=100$.

\subsection{Extinction Correction}

The logarithmic extinction parameter at $\mathrm{H} \beta, c(\mathrm{H} \beta)$, was derived for the three M31 PNe by comparing the observed $\mathrm{H}$ I Balmer line ratio, $I(\mathrm{H} \gamma) / I(\mathrm{H} \delta)$, with the predicted Case $\mathrm{B}$ value. This methodology yields a $c(\mathrm{H} \beta)$ value of $0.43,0.51$, and 0.54 for PN1, PN2, and PN3, respectively. The $\mathrm{H}_{\mathrm{I}} \lambda 3889$ $(n=2-8)$ and $\lambda 3970(n=2-7)$ lines were not used for the extinction correction because the former line is blended with He I $\lambda 3888\left(2 s{ }^{3} S-3 p{ }^{3} P^{\mathrm{o}}\right)$ and the latter line is blended with the [Ne III] $\lambda 3967\left(2 p^{4}{ }^{3} P_{1}-{ }^{1} D_{2}\right)$ line. The observed line fluxes were dereddened by

$$
I(\lambda)=10^{c(\mathrm{H} \beta) \mathrm{f}(\lambda)} F(\lambda),
$$



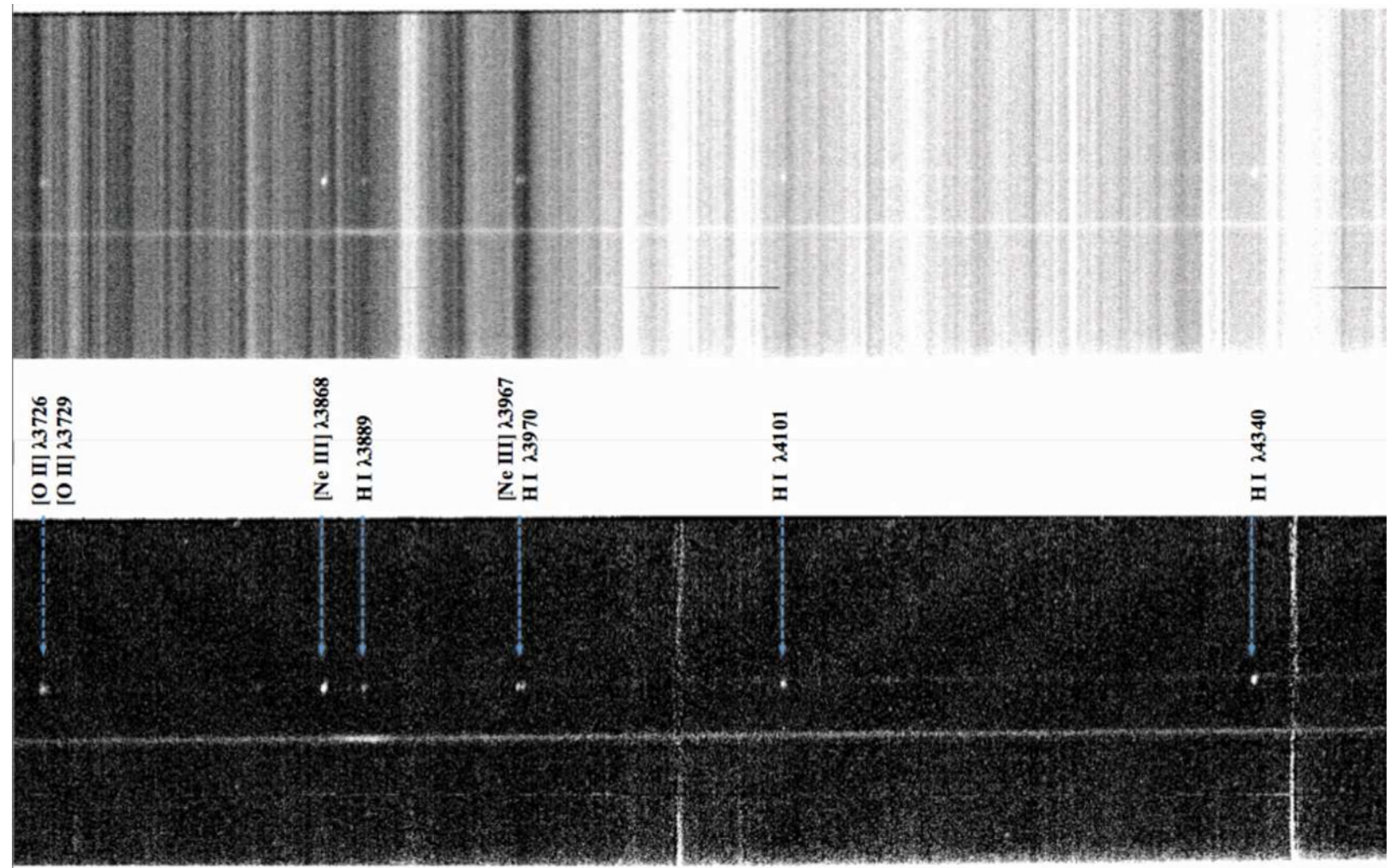

Figure 5. Same as Figure 4 but for PN2. The [O III] $\lambda 4363$ auroral line is not seen in the sky-subtracted spectrum (the lower panel). A foreground star is located about 18 arcsec below PN2.

(A color version of this figure is available in the online journal.)

where $f(\lambda)$ is the extinction curve (relative to the $f$ value at $\mathrm{H} \beta$ ) adopted from Cardelli et al. (1989) with a total-to-selective extinction ratio $R_{\mathrm{V}}=3.1$.

The method of extinction correction described above assumes that the Galactic foreground extinction is negligible compared to the local extinction in M31. In order to assess how important the Galactic foreground extinction can be to the line fluxes, we carried out another reddening correction procedure. We first corrected for the MW foreground extinction and then we corrected for the local extinction. The foreground reddening $E(B-V)$ toward M31 is 0.062 , which was adopted from Schlegel et al. (1998). These authors conducted an all-sky survey of infrared dust emission to calculate the reddening $E(B-V)$ across the sky to within an uncertainty of $\sim 16 \%$. After the MW foreground extinction toward M31 had been corrected for, we used the $\mathrm{H}$ I $I(\mathrm{H} \alpha) / I(\mathrm{H} \beta)$ ratio to correct for the local extinction. The derived logarithmic extinction parameter $c(\mathrm{H} \beta)$ of $\mathrm{PN} 1$, $\mathrm{PN} 2$, and PN3 is $0.30,0.31$, and 0.45 , respectively.

The extinction-corrected line fluxes based on the above two methods differ by $\sim 10 \%$. Since the correction for the MW foreground extinction may introduce extra uncertainties and since so far most observations of the M31 nebulae (PNe and $\mathrm{H}$ II regions) have only been corrected for extinction once (e.g., Jacoby \& Ciardullo 1999; Kwitter et al. 2012; Sanders et al. 2012; Zurita \& Bresolin 2012) using a certain extinction law, we adopted the first set of the extinction parameters in our data analysis (Table 2). If the same extinction law (e.g., Cardelli et al. 1989) is used, we would expect that the MW and M31 extinctions could be "packed" into a single $c(\mathrm{H} \beta)$, which is equivalent to the result of the first method.

\section{RESULTS}

\subsection{Relative Line Intensities}

The extinction-corrected relative line intensities of the three PNe are presented in Table 2. As mentioned in Section 2.3, the $\mathrm{H} \gamma$ line was used for flux normalization of the blue data and $\mathrm{H} \alpha$ was used for flux normalization of the red data. Here, the Balmer line ratios $I(\mathrm{H} \gamma) / I(\mathrm{H} \beta)$ and $I(\mathrm{H} \alpha) / I(\mathrm{H} \beta)$ were adopted from Case B theoretical calculations. The assumed electron temperature and density are valid because the H I Balmer line ratios are mostly insensitive to these two physical quantities. Continua were not clearly detected in the spectra due to the faintness of the objects. Thus, the integrated line fluxes were directly obtained from Gaussian profile fits. As an example, Figure 7 shows Gaussian profile fits to the [O II] and [O III] lines detected in the blue spectra of the three PNe. Uncertainties in the line intensities were estimated from direct integration and the Gaussian profile fitting of the profile of each emission line. PN3 has the relatively highest $\mathrm{S} / \mathrm{N}$ blue spectra among the three PNe. For example, the $\mathrm{S} / \mathrm{N}$ of the $\mathrm{H} \gamma \lambda 4340$ line in the blue spectrum of PN3 is 23.4, while PN1 and PN2 have ratios of 9.8 and 9.5 , respectively. The differences in the data quality of the blue spectra of the three PNe can be seen in Figure 2.

Although the $\mathrm{Hg}$ I $\lambda 4358$ mercury line at the Palomar Observatory is bright, it was expected that the [O III] $\lambda 4363$ line 

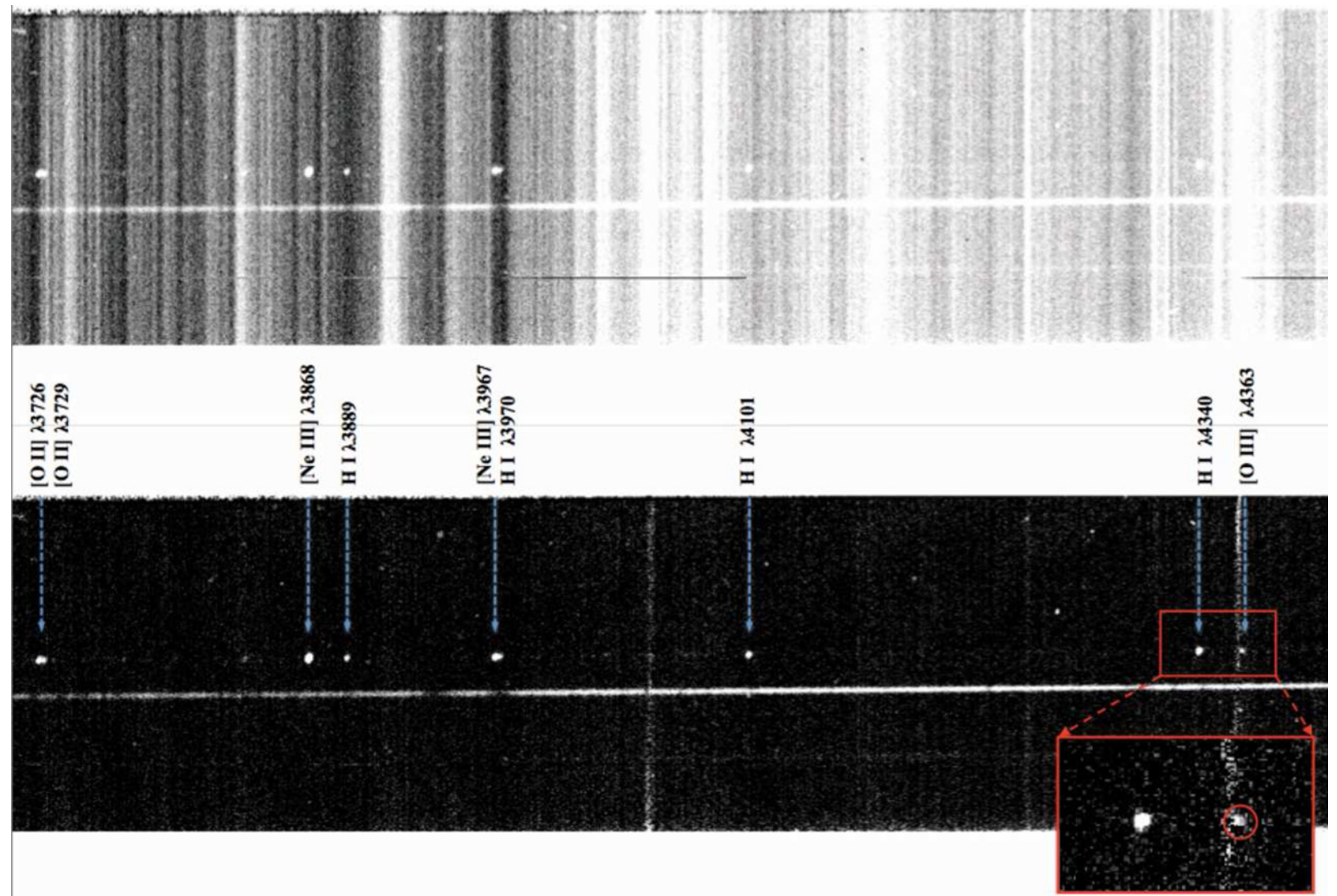

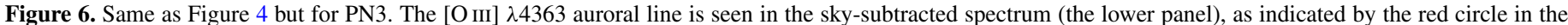
inset. A bright foreground star is located about 13 arcsec below PN3.

(A color version of this figure is available in the online journal.)

would be detected in all three $\mathrm{PNe}$, given the radial velocities of the three objects (Table 1), if the subtraction of the sky background was well done. However, the $\left[\mathrm{O}_{\mathrm{III}}\right] \lambda 4363$ line was not detected in the blue spectrum of PN2 (Figures 5 and 7); this object has the medium radial velocity $\left(-44.4 \mathrm{~km} \mathrm{~s}^{-1}\right)$ of the three objects, probably due to the relatively low $\mathrm{S} / \mathrm{N}$. The [O III] $\lambda 4363$ line was detected in PN1 (Figures 4 and 7), which has the lowest radial velocity $\left(-19.6 \mathrm{~km} \mathrm{~s}^{-1}\right)$, with an $\mathrm{S} / \mathrm{N}$ of 5.3. The $\mathrm{S} / \mathrm{N}$ of the blue spectrum of PN3 is higher than that of the other two objects. Therefore, although the radial velocity of PN3 is the highest among the three PNe $\left(-90.3 \mathrm{~km} \mathrm{~s}^{-1}\right)$, a weak [O III] $\lambda 4363$ line was still clearly detected (Figures 6 and 7), with an $\mathrm{S} / \mathrm{N}$ of $\sim 4.0$, which is close to that of PN1. Although much effort was made to subtract the sky background (see Section 2.3), accurate measurements of the [O III] $\lambda 4363$ line for our PN sample are still affected by the residuals of sky subtraction, as can be seen in Figures 4 and 6. Integrated fluxes of the [O III] $\lambda 4363$ line of PN1 and PN3 are given in Table 2, and the uncertainties in the fluxes were estimated from Gaussian profile fitting (Figure 7). Electron temperature diagnostics for PN1 and PN3 using the $[\mathrm{O}$ III $](\lambda 4959+\lambda 5007) / \lambda 4363$ line ratio are presented in Section 3.2.

The [O II] $\lambda \lambda 3726,3729$ lines are detected in the blue spectrum of PN3. Two Gaussian profiles were used to fit the doublet (Figure 7$)$, which yields an $I(\lambda 3726) / I(\lambda 3729)$ intensity ratio of 1.4. Measurement uncertainties of the two [O II] lines for PN3 are $\sim 20 \%-30 \%$. The double-peak profile of the [O II] doublet lines in the spectrum of PN2 is not so obvious as in PN3; the data of PN3 are of higher quality. However, Gaussian profile fitting yields an $I(\lambda 3726) / I(\lambda 3729)$ ratio of 1.5 for PN2, which is quite reasonable. Uncertainties in the line fluxes of the [O II] lines of PN2 could be $\sim 30 \%-40 \%$. The [O II] line ratio observed in PN1 is 2.8. Electron densities are estimated for the three $\mathrm{PNe}$ using the [O II] line ratio in Section 3.2.

Some important emission lines detected suffer from blending. The [Ne III] $\lambda 3967$ line is blended with H I $\lambda 3970$. We corrected for the flux contribution from the H I $\lambda 3970$ line using the theoretical Hi $I(\lambda 3970) / I(\lambda 4340)$ ratio. The $\mathrm{Ne}^{2+} / \mathrm{H}^{+}$abundances were then derived from the corrected [Ne III] $\lambda 3967$ line flux. The Hi $\lambda 3889$ line is blended with the He I $2 s{ }^{3} S-3 p^{3} P^{\mathrm{o}} \lambda 3888$ line (Table 2). We corrected the $\mathrm{He}$ I line flux for the contribution from the $\mathrm{H}$ I $\lambda 3889$ line, using the observed flux of the $\mathrm{HI} \lambda 4340$ line and the theoretical $\mathrm{H}_{\mathrm{I}}(\lambda 3889) / I(\lambda 4340)$ ratio. The corrected fluxes of the $\mathrm{He}$ I $\lambda 3888$ line were used to derive the $\mathrm{He}^{+} / \mathrm{H}^{+}$abundance for the three PNe (Section 3.3). The [S II] $\lambda 6731\left(3 p^{3}{ }^{4} S_{3 / 2}^{\mathrm{o}}{ }^{2} D_{3 / 2}^{\mathrm{o}}\right)$ line is detected in the red spectrum of PN3 (Figure 3), with an $\mathrm{S} / \mathrm{N}$ of $\sim 3-4$. The other component of the [S II] doublet, the $\lambda 6716\left(3 p^{3}{ }^{4} S_{3 / 2}^{\mathrm{o}}-{ }^{2} D_{5 / 2}^{\mathrm{o}}\right)$ line, which is expected to be weaker than the $\lambda 6731$ line, is not clearly seen due to its weakness. The [Ar III] $\lambda 7136\left(3 p^{4}{ }^{3} P_{2}{ }^{1} D_{2}\right)$ line is also detected in the spectrum of PN3. The $\mathrm{S}^{+} / \mathrm{H}^{+}$and $\mathrm{Ar}^{2+} / \mathrm{H}^{+}$ionic abundances are derived from the $\lambda 6731$ and $\lambda 7136$ lines, respectively, in Section 3.3. 
Table 2

Emission Lines Detected in the Spectra of the Three Northern Spur PNe

\begin{tabular}{|c|c|c|c|c|c|}
\hline \multirow[t]{2}{*}{ Ion } & \multirow{2}{*}{$\begin{array}{c}\lambda \\
(\AA)\end{array}$} & \multirow[t]{2}{*}{ Transition } & \multicolumn{3}{|c|}{$I(\lambda)$} \\
\hline & & & PN1 & $\mathrm{PN} 2$ & PN3 \\
\hline [O II $]$ & 3726 & $2 p^{3}{ }^{4} S_{3 / 2}^{\mathrm{o}}-2 p^{3}{ }^{2} D_{3 / 2}^{\mathrm{o}}$ & $50 \pm 10$ & $58 \pm 14$ & $43 \pm 8$ \\
\hline [O II $]$ & 3729 & $2 p^{3}{ }^{4} S_{3 / 2}^{\mathrm{o}}-2 p^{3}{ }^{2} D_{5 / 2}^{\mathrm{o}}$ & $17.8 \pm 6.5$ & $42 \pm 12$ & $29 \pm 6$ \\
\hline [Ne III] & 3868 & $2 p^{4}{ }^{3} P_{2}-2 p^{4}{ }^{1} D_{2}$ & $123 \pm 11$ & $94 \pm 10$ & $136 \pm 5$ \\
\hline $\mathrm{HI}$ & 3889 & $2 p^{2} P^{\mathrm{o}}-8 d^{2} D$ & $24 \pm 3$ & $21 \pm 4$ & $21 \pm 3$ \\
\hline [Ne III $]$ & 3967 & $2 p^{4}{ }^{3} P_{1}-2 p^{4}{ }^{1} D_{2}$ & $53 \pm 9$ & $45 \pm 6$ & $56 \pm 4$ \\
\hline $\mathrm{HI}_{\mathrm{I}}$ & 4101 & $2 p^{2} P^{\mathrm{o}}-6 d^{2} D$ & $30 \pm 5$ & $35 \pm 7$ & $25 \pm 2$ \\
\hline $\mathrm{HI}_{\mathrm{I}}$ & 4340 & $2 p^{2} P^{\mathrm{o}}-5 d^{2} D$ & $47 \pm 8$ & $47 \pm 7$ & $47 \pm 4$ \\
\hline [O III $]$ & 4363 & $2 p^{2}{ }^{1} D_{2}-2 p^{2}{ }^{1} S_{1}$ & $14.5 \pm 4.4$ & & $8.3 \pm 2.5$ \\
\hline $\mathrm{He}$ I & 4471 & $4 d^{3} D-2 p^{3} P^{\mathrm{o}}$ & $8.8 \pm 2.9$ & & $7.1 \pm 2.2$ \\
\hline $\mathrm{HI}^{\mathrm{a}}$ & 4861 & $2 p^{2} P^{o}-4 d^{2} D$ & 100 & 100 & 100 \\
\hline [O III $]$ & 4959 & $2 p^{2}{ }^{3} P_{1}-2 p^{2}{ }^{1} D_{2}$ & $406 \pm 24$ & $302 \pm 20$ & $460 \pm 7$ \\
\hline [O III] & 5007 & $2 p^{2}{ }^{3} P_{2}-2 p^{2}{ }^{1} D_{2}$ & $1075 \pm 19$ & $875 \pm 18$ & $1238 \pm 6$ \\
\hline He I & 5876 & $2 p^{3} P^{\mathrm{o}}-3 d^{3} D$ & & $12.8 \pm 3.9$ & $11.9 \pm 2.3$ \\
\hline$\left[\mathrm{N}_{\mathrm{II}}\right]$ & 6548 & $2 p^{2}{ }^{3} P_{1}-2 p^{2}{ }^{1} D_{2}$ & $11 \pm 3$ & $31 \pm 9$ & $25 \pm 5$ \\
\hline $\mathrm{HI}_{\mathrm{I}}$ & 6563 & $2 p^{2} P^{\mathrm{o}}-3 d^{2} D$ & $285 \pm 7$ & $285 \pm 8$ & $285 \pm 2$ \\
\hline$[\mathrm{N}$ II $]$ & 6583 & $2 p^{2}{ }^{3} P_{2}-2 p^{2}{ }^{1} D_{2}$ & $44 \pm 7$ & $77 \pm 10$ & $65 \pm 3$ \\
\hline He I & 6678 & $2 p^{1} P^{\mathrm{O}}-3 d^{1} D$ & & & $5.0 \pm 2.7$ \\
\hline$[\mathrm{S} \mathrm{II}]$ & 6731 & $2 p^{3}{ }^{4} S_{3 / 2}^{\mathrm{o}}-2 p^{3}{ }^{2} D_{3 / 2}^{\mathrm{o}}$ & & & $6.1 \pm 2.9$ \\
\hline He I & 7065 & $2 p^{3} P^{\mathrm{o}}-3 s^{3} S$ & $7.8 \pm:$ & $12.1 \pm:$ & $6.2 \pm:$ \\
\hline [Ar III] & 7136 & $3 p^{43} P_{2}-3 p^{4}{ }^{1} D_{2}$ & & & $13 \pm 3$ \\
\hline$c(\mathrm{H} \beta)$ & & & 0.43 & 0.51 & 0.54 \\
\hline
\end{tabular}

Notes. All intensities have been corrected for extinction. For the blue spectra (3400-4800 $\AA$ ), line intensities are normalized such that $I(\mathrm{H} \gamma)=47$; for the red spectra (4700-7300 $\AA$ ), line intensities are normalized such that $I(\mathrm{H} \alpha)=$ 285. A colon ":" denotes that the uncertainty in line flux is large $(>100 \%)$.

a The integrated flux of $\mathrm{H} \beta$ is assumed to be 100 .

\subsection{Plasma Diagnostics}

Plasma diagnostics were carried out using the collisionally excited lines (CELs) of heavy elements (e.g., Osterbrock \& Ferland 2006) detected in the spectra of our PN sample. The [O II] $\lambda 3726 / \lambda 3729$ line ratio was used to determine the electron density and the $\left[\mathrm{O}_{\mathrm{III}}\right](\lambda 4959+\lambda 5007) / \lambda 4363$ nebular-to-auroral line ratio was used to determine the electron temperature. The results of the plasma diagnostics are given in Table 3.

Figure 8 shows the plasma diagnostic diagrams of the three PNe. These diagrams were created by solving the level population equations for five-level atomic models using the program EQUIB. ${ }^{13}$ The $[\mathrm{O}$ III $](\lambda 4959+\lambda 5007) / \lambda 4363$ nebularto-auroral and the $\left[\mathrm{O}_{\mathrm{II}}\right] \lambda 3726 / \lambda 3729$ nebular line ratios observed in PN1 yield an electron temperature of $12,200 \mathrm{~K}$ and a density of $\sim 12,800 \mathrm{~cm}^{-3}$, respectively. The [O III] and [O II] line ratios observed in the spectrum of $\mathrm{PN} 3$ yield an electron temperature of $10,080 \mathrm{~K}$ and a density of $\sim 1650 \mathrm{~cm}^{-3}$, respectively. Since the [O III] $\lambda 4363$ line was not detected in the spectrum of PN2 (Figure 7), an electron temperature of $10^{4} \mathrm{~K}$, a typical forbidden-line temperature of $\mathrm{PNe}$, was assumed for this object. At this temperature, an electron density of $\sim 1450 \mathrm{~cm}^{-3}$ was derived for PN2 from the observed [O II] line ratio.

The derived electron density of PN1 is significantly higher than that of PN2 and PN3 and its temperature is also slightly higher. These results indicate that PN1 may be a relatively younger (compact) PN compared to the other two objects. However, the electron density derived for PN1 may have a relatively

\footnotetext{
13 EQUIB was originally developed by I. D. Howarth et al. from the Department of Physics and Astronomy, University College London, for calculating the level population equations of multi-level $(n \geqslant 5)$ atomic models.
}

Table 3

Plasma Diagnostics

\begin{tabular}{lccc}
\hline \hline Diagnostic Ratio & PN1 & PN2 & PN3 \\
\hline [O III $](\lambda 4959+\lambda 5007) / \lambda 4363$ & $12200 \pm 1200$ & $\begin{array}{c}T_{\mathrm{e}}(\mathrm{K}) \\
10000^{\mathrm{a}}\end{array}$ & $10080 \pm 800$ \\
& & $N_{\mathrm{e}}\left(\mathrm{cm}^{-3}\right)$ & \\
{$\left[\mathrm{O}_{\text {II }}\right] \lambda 3726 / \lambda 3729$} & $12800 \pm 3800$ & $1450 \pm 500$ & $1650 \pm 300$ \\
\hline
\end{tabular}

Note. ${ }^{a}$ An assumed electron temperature for PN2 because its [O III] $\lambda 4363$ line is not observed.

large uncertainty, given the faintness of the [O II] $\lambda 3729$ line. In order to check how much the electron density would affect the resultant ionic abundances, we carried out abundance calculations for two density cases, $N_{\mathrm{e}}=10^{3}$ and $10^{4} \mathrm{~cm}^{-3}$, at $T_{\mathrm{e}}=12,200 \mathrm{~K}$. The ionic abundances derived from the [O III] and [Ne III] CELs at the two density cases differ by only $3 \%$, and the ionic abundances derived from the [N $\mathrm{NI}]$ nebular lines differ by about $10 \%$ at these two densities; that difference increases to nearly $40 \%$ for the $\mathrm{O}^{+} / \mathrm{H}^{+}$ionic abundances derived from the [O II] nebular lines. The above differences in the ionic abundances at the two densities are expected because of the large differences in critical densities: $:^{14}$ the critical densities of the [O III] and [Ne III] nebular lines (which have the same upper level ${ }^{1} D_{2}$ for each ion) are $6.8 \times 10^{5}$ and $9.5 \times 10^{6} \mathrm{~cm}^{-3}$, respectively, which are much higher than the typical PN densities. The critical density of the $[\mathrm{N}$ II] $\lambda \lambda 6548,6583$ nebular lines (which also have the same upper level ${ }^{1} D_{2}$ ) is $6.6 \times 10^{4} \mathrm{~cm}^{-3}$, which is also relatively higher than the average PN density; the critical densities of the $[\mathrm{O}$ II] $\lambda \lambda 3726,3729$ nebular lines (whose upper levels are the ${ }^{2} D_{3 / 2}^{\mathrm{o}}$ and ${ }^{2} D_{5 / 2}^{\mathrm{o}}$ fine-structure levels, respectively) are $1.5 \times 10^{4}$ and $3400 \mathrm{~cm}^{-3}$, respectively, which are comparable to the typical PN densities. It can be seen from the diagnostic diagrams of PN1 and PN3 (Figure 8) that the [O III] line ratio is very sensitive to temperature, while varying the electron density does not change the resultant temperature very much.

\subsection{Ionic and Elemental Abundances}

Ionic abundances derived from the emission lines detected in the spectra of the three PNe are presented in Table 4. In order to derive ionic abundances from CELs, the equations of statistical equilibrium were solved using the program EQUIB to derive the population of the upper level of a transition. The electron temperatures and densities given in Table 3, which were obtained from plasma diagnostics (Section 3.2), were assumed for abundance determinations.

Several He I lines are detected. The $\lambda 3888$ line is blended

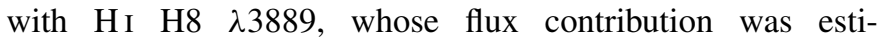
mated from the observed $\mathrm{HI}_{\mathrm{I}} \lambda 4340$ line and the theoretical $\mathrm{H} \mathrm{I} I(\lambda 3889) / I(\lambda 4340)$ ratio at $T_{\mathrm{e}}=10,000 \mathrm{~K}$ and $N_{\mathrm{e}}=10^{4} \mathrm{~cm}^{-3}$ in Case B. The flux-corrected He I $\lambda 3888$ line yields $\mathrm{He}^{+} / \mathrm{H}^{+}$ ionic abundances that are generally lower than the other He I lines, $\lambda \lambda 4471,5876$, and 6678 , which are too weak to be accurately measured. However, the $\mathrm{He}^{+} / \mathrm{H}^{+}$abundance ratio derived from the $\lambda 5876$ line observed in PN3 agrees with that derived from the $\lambda 3888$ line. The effective recombination coefficients for the He I spectrum calculated by Benjamin et al. (1999) were used for the abundance determinations. The $\lambda \lambda 3888$ and 5876 lines are among the strongest He I optical recombination lines observed in PNe. At $T_{\mathrm{e}}=10,000 \mathrm{~K}$ and $N_{\mathrm{e}}=10^{4} \mathrm{~cm}^{-3}$, the

\footnotetext{
$\overline{14}$ Critical densities are all quoted for an electron temperature of $10^{4} \mathrm{~K}$.
} 

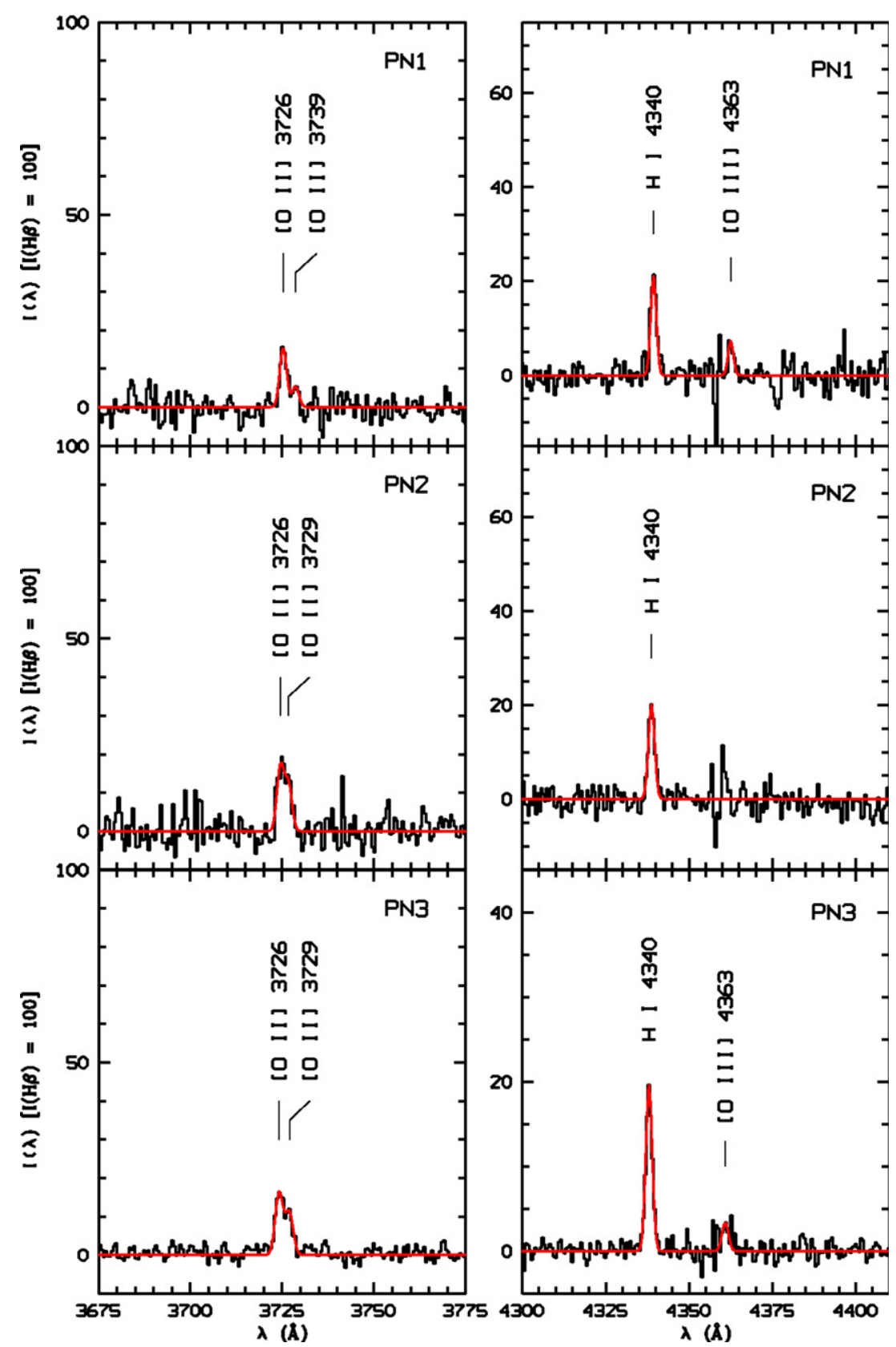

Figure 7. Blue spectra of PN1 (top panel), PN2 (middle panel), and PN3 (bottom panel), showing the observed [O II] and [O III] lines. The red continuous curves are Gaussian profile fits to the [O II] $\lambda \lambda 3726,3729$ doublet (the left column) and the [O III] $\lambda 4363$ line (the right column). The [O III] $\lambda 4363$ line is not detected in PN2, and the feature at $4360 \AA$ in its blue spectrum (the middle-right panel) is likely to be a residual from sky subtraction. The spectra have been normalized such that $\mathrm{H} \beta$ has an integrated flux of 100 and extinction has been corrected for.

(A color version of this figure is available in the online journal.)

$I(\lambda 3888) / I(\lambda 4471)$ and $I(\lambda 5876) / I(\lambda 4471)$ ratios of He I are 2.6 and 2.9 , respectively, while the intensities of the other He I optical recombination lines relative to $\lambda 4471$ are all less than 0.95 (Benjamin et al. 1999). That pattern of the relative intensities of the He I optical lines remains when the physical conditions change from $T_{\mathrm{e}}=5000 \mathrm{~K}$ and $N_{\mathrm{e}}=10^{2} \mathrm{~cm}^{-3}$ to $T_{\mathrm{e}}=20,000 \mathrm{~K}$ and $N_{\mathrm{e}}=10^{6} \mathrm{~cm}^{-3}$. The reason that we chose $T_{\mathrm{e}}=5000$ and $20,000 \mathrm{~K}$ as the lower and upper limits of the physical conditions is that, so far, the electron temperatures of PNe derived from the $\mathrm{He}$ I recombination line ratios largely lie in the range 5000-12,000 K (e.g., Zhang et al. 2005). Given that the $\mathrm{H}_{\mathrm{I}}$ Balmer line ratios are mostly insensitive to temperature and density, the line flux of $\mathrm{He}$ I $\lambda 3888$ corrected for the contribution from the blended $\mathrm{H}_{\mathrm{I}} \lambda 3889$ line is reliable, probably better than $20 \%$. Although the strength of the He I $\lambda 3888$ line could be significantly affected by the effect of self-absorption due to a large population in the $2{ }^{3} S$ metastable level (e.g., Robbins 1968; Benjamin et al. 2002), the majority of the line flux uncertainty is contributed by measurement errors.

Although the [O III] $\lambda 4363$ lines detected in the blue spectra of PN1 and $\mathrm{PN} 3$ are faint $(\mathrm{S} / \mathrm{Ns} \sim 4-5)$, the $\mathrm{O}^{2+} / \mathrm{H}^{+}$abundance derived from this line agrees with that derived from the [O III] $\lambda \lambda 4959$ and 5007 nebular lines within the errors. This result was expected because the abundances were derived assuming the electron temperature yielded by the [O III] line ratio. The three [O III] lines detected in the spectra of both PN1 and PN3 

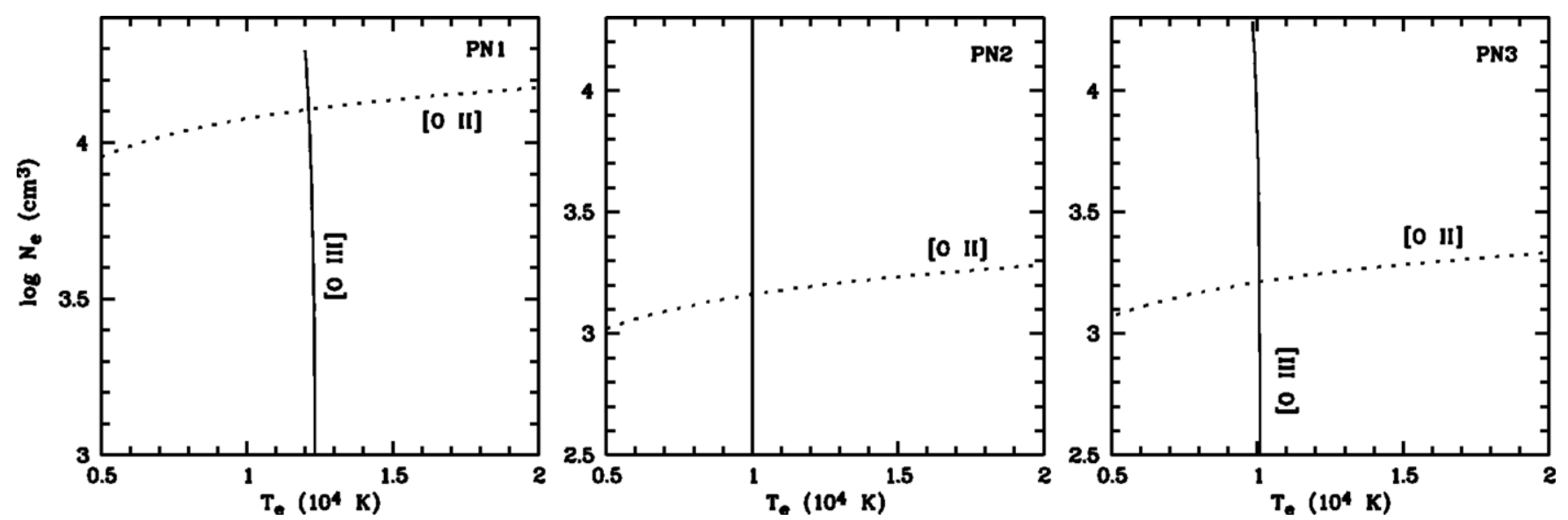

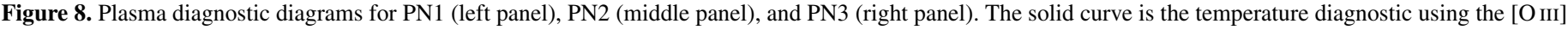

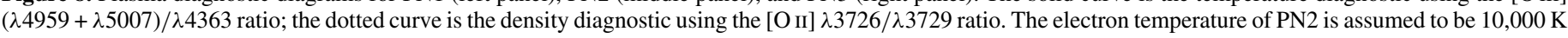
because the $[\mathrm{O} \mathrm{III}] \lambda 4363$ line is not detected in the spectrum of this object.

Table 4

Ionic Abundances

\begin{tabular}{|c|c|c|c|c|}
\hline \multirow[t]{2}{*}{ Ion } & \multirow{2}{*}{$\begin{array}{l}\text { Line } \\
(\AA)\end{array}$} & \multicolumn{3}{|c|}{ Abundance $\left(\mathrm{X}^{i+} / \mathrm{H}^{+}\right)$} \\
\hline & & PN1 & PN2 & PN3 \\
\hline \multirow[t]{4}{*}{$\mathrm{He}^{+}$} & $\lambda 3888$ & $0.106( \pm 0.027)$ & $0.090( \pm 0.020)$ & $0.097( \pm 0.015)$ \\
\hline & $\lambda 4471$ & $0.149( \pm 0.074)$ & & $0.146( \pm 0.044)$ \\
\hline & $\lambda 5876$ & & $0.133( \pm 0.039)$ & $0.095( \pm 0.023)$ \\
\hline & $\lambda 6678$ & & & $0.129( \pm 0.058)$ \\
\hline Adopted $^{\mathrm{a}}$ & & $0.106( \pm 0.027)$ & $0.090( \pm 0.020)$ & $0.097( \pm 0.015)$ \\
\hline \multirow[t]{2}{*}{$\mathrm{N}^{+}$} & $\lambda 6548$ & $3.9( \pm 1.1) \times 10^{-6}$ & $8.4( \pm 2.1) \times 10^{-6}$ & $1.2( \pm 0.5) \times 10^{-5}$ \\
\hline & $\lambda 6583$ & $5.4( \pm 1.0) \times 10^{-6}$ & $7.1( \pm 0.9) \times 10^{-6}$ & $1.1( \pm 0.1) \times 10^{-5}$ \\
\hline Adopted $^{b}$ & & $5.4( \pm 1.0) \times 10^{-6}$ & $7.1( \pm 0.9) \times 10^{-6}$ & $1.1( \pm 0.1) \times 10^{-5}$ \\
\hline \multirow[t]{2}{*}{$\mathrm{O}^{+}$} & $\lambda 3726$ & $3.3( \pm 0.7) \times 10^{-5}$ & $2.3( \pm 0.4) \times 10^{-5}$ & $3.3( \pm 0.6) \times 10^{-5}$ \\
\hline & $\lambda 3729$ & $2.9( \pm 1.1) \times 10^{-5}$ & $2.3( \pm 0.5) \times 10^{-5}$ & $3.4( \pm 0.7) \times 10^{-5}$ \\
\hline Adopted $^{c}$ & & $3.3( \pm 0.4) \times 10^{-5}$ & $2.3( \pm 0.4) \times 10^{-5}$ & $3.3( \pm 0.6) \times 10^{-5}$ \\
\hline \multirow[t]{3}{*}{$\mathrm{O}^{2+}$} & $\lambda 4363$ & $2.6( \pm 0.7) \times 10^{-4}$ & & $4.4( \pm 0.9) \times 10^{-4}$ \\
\hline & $\lambda 4959$ & $2.9( \pm 0.2) \times 10^{-4}$ & $1.8( \pm 0.1) \times 10^{-4}$ & $4.8( \pm 0.1) \times 10^{-4}$ \\
\hline & $\lambda 5007$ & $2.8( \pm 0.2) \times 10^{-4}$ & $1.8( \pm 0.1) \times 10^{-4}$ & $4.3( \pm 0.1) \times 10^{-4}$ \\
\hline Adopted $^{\mathrm{d}}$ & & $2.8( \pm 0.2) \times 10^{-4}$ & $1.8( \pm 0.1) \times 10^{-4}$ & $4.5( \pm 0.1) \times 10^{-4}$ \\
\hline \multirow[t]{2}{*}{$\mathrm{Ne}^{2+}$} & $\lambda 3868$ & $5.8( \pm 0.5) \times 10^{-5}$ & $3.5( \pm 0.4) \times 10^{-5}$ & $1.3( \pm 0.1) \times 10^{-4}$ \\
\hline & $\lambda 3967$ & $5.6( \pm 0.9) \times 10^{-5}$ & $3.5( \pm 0.5) \times 10^{-5}$ & $1.3( \pm 0.1) \times 10^{-4}$ \\
\hline Adopted $^{\mathrm{e}}$ & & $5.8( \pm 0.6) \times 10^{-5}$ & $3.5( \pm 0.4) \times 10^{-5}$ & $1.3( \pm 0.1) \times 10^{-4}$ \\
\hline $\mathrm{S}^{+}$ & $\lambda 6731$ & & & $3.1( \pm 1.0) \times 10^{-7}$ \\
\hline $\mathrm{Ar}^{2+}$ & $\lambda 7136$ & & & $1.1( \pm 0.3) \times 10^{-6}$ \\
\hline
\end{tabular}

Notes.

a The $\mathrm{He}^{+} / \mathrm{H}^{+}$abundance ratio derived from the $\lambda 3888$ line is adopted.

b The $\mathrm{N}^{+} / \mathrm{H}^{+}$abundance derived from the $[\mathrm{N} \mathrm{II}] \lambda 6583$ line is adopted.

${ }^{c}$ For PN1, the $\mathrm{O}^{+} / \mathrm{H}^{+}$abundance derived from the $\lambda 3726$ line is adopted.

d The adopted $\mathrm{O}^{2+} / \mathrm{H}^{+}$abundance is derived from the total flux of [O $\left.\mathrm{III}\right] \lambda \lambda 4959$, 5007.

e $\mathrm{The}^{2+} / \mathrm{H}^{+}$abundance derived from the $[\mathrm{Ne} \mathrm{III}] \lambda 3868$ line is adopted.

yield consistent $\mathrm{O}^{2+} / \mathrm{H}^{+}$ionic abundances. The electron temperature of PN1 derived from the [O III] $\lambda 4363$ line is slightly higher than that of PN3. It is known that the weakly detected lines tend to be overmeasured when an accurate sky subtraction is difficult (Kwitter et al. 2012). Considering the fact that the radial velocity of PN1 is lower than that of PN3, and consequently an accurate subtraction of the $\lambda 4358$ mercury line for PN3 is expected to be more difficult than for PN1, the relatively higher electron temperature derived for PN1 indicates that the intensity of the [O III] $\lambda 4363$ line of PN1 might be overestimated. Thus, we adopted an abundance ratio of $2.8 \times 10^{-4}$, which was calculated from the total intensity of the [O III] $\lambda 4959$ and 5007 lines, as the $\mathrm{O}^{2+} / \mathrm{H}^{+}$abundance for PN1. For PN3, that abundance ratio is $4.5 \times 10^{-4}$. The total intensity of the [O III] $\lambda \lambda 4959$ and 5007 lines in PN2 yields an abundance ratio of $1.8 \times 10^{-4}$.

In both $\mathrm{PN} 2$ and $\mathrm{PN} 3$, the $\mathrm{O}^{+} / \mathrm{H}^{+}$ionic abundances derived from the $\lambda \lambda 3726$ and 3729 lines agree with each other, which was expected because the ionic abundances were determined based on the electron density yielded by the [O II] $\lambda 3726 / \lambda 3729$ line ratio. For PN1, the ionic abundances derived from the [O II] lines agree within the errors, although slight differences are present, mainly due to the relatively large measurement uncertainty in the $\lambda 3729$ line. The $\mathrm{O}^{+} / \mathrm{H}^{+}$abundance ratio derived from the $\lambda 3726$ line was adopted for all PNe. The $\mathrm{N}^{+} / \mathrm{H}^{+}$abundances derived from the $\left[\mathrm{N}_{\mathrm{II}}\right] \lambda \lambda 6548$ and 6583 nebular lines detected in the red spectrum of PN1 differ by about $40 \%$, while in PN2 and PN3 the abundances differ by $19 \%$ and $10 \%$, respectively. Such a significant difference in PN1 is due to a relatively large uncertainty in the faint $[\mathrm{N} \mathrm{II}] \lambda 6548$ line. Uncertainties in the $\mathrm{N}^{+} / \mathrm{H}^{+}$ionic abundances of $\mathrm{PN} 3$ are smaller because its red spectrum is of higher quality than that of the other two PNe. We adopted the abundances calculated from the stronger $\lambda 6583$ line as the $\mathrm{N}^{+} / \mathrm{H}^{+}$ionic abundances for the three PNe.

The [Ne III] $\lambda 3967$ line is blended with Hi H7 $\lambda 3970$, whose flux contribution was estimated from the observed $\lambda 4340$ line and the theoretical $\mathrm{H}_{\mathrm{I}} \lambda 3970 / \lambda 4340$ ratio. For all three $\mathrm{PNe}$, the corrected flux of the [Ne III] $\lambda 3967$ line yields an $\mathrm{Ne}^{2+} / \mathrm{H}^{+}$ionic abundance that is generally consistent with the [Ne III] $\lambda 3868$ line. Given that the $\lambda 3868$ line is stronger, the $\mathrm{Ne}^{2+} / \mathrm{H}^{+}$abundance derived from this [Ne III] line is adopted for all three objects. The $\mathrm{S}^{+} / \mathrm{H}^{+}$and $\mathrm{Ar}^{2+} / \mathrm{H}^{+}$abundance ratios were derived from the [S II] $\lambda 6731$ and [Ar III] $\lambda 7136$ lines observed in the red spectrum of PN3, respectively (Table 4).

The uncertainties of the abundances that appear in the brackets following the abundance ratios in Table 4 were estimated based on two sources: (1) the measurement uncertainties of line fluxes and (2) the uncertainties in electron temperatures resulting from the measurement uncertainties of the [O III] $\lambda 4363$ auroral line. For PN1 and PN3, where the [O III] $\lambda 4363$ line was detected in the spectra, uncertainties in the ionic abundances of heavy elements mainly arise from the second source. This fact is because the emissivities of the heavy element CELs are very sensitive to electron temperature under nebular conditions (e.g., 
Table 5

Elemental Abundances ${ }^{\mathrm{a}}$

\begin{tabular}{lllllll}
\hline \hline \multirow{2}{*}{ Element } & \multicolumn{5}{c}{ X/H } \\
\cline { 2 - 7 } & \multicolumn{2}{c}{ PN1 } & \multicolumn{2}{c}{ PN2 } & \multicolumn{2}{c}{ PN3 } \\
\hline $\mathrm{N}$ & $8.7( \pm 1.8) \times 10^{-5}$ & 7.94 & $1.0( \pm 0.4) \times 10^{-4}$ & 8.01 & $1.7( \pm 0.3) \times 10^{-4}$ & 8.23 \\
$\mathrm{O}$ & $3.6( \pm 0.5) \times 10^{-4}$ & 8.55 & $2.6( \pm 0.5) \times 10^{-4}$ & 8.42 & $5.2( \pm 0.6) \times 10^{-4}$ & 8.72 \\
$\mathrm{Ne}$ & $7.4( \pm 1.4) \times 10^{-5}$ & 7.87 & $5.8( \pm 1.4) \times 10^{-5}$ & 7.76 & $1.5( \pm 0.3) \times 10^{-4}$ & 8.16 \\
$\mathrm{~S}$ & & & & & $4.8( \pm 2.1) \times 10^{-6}$ & 6.68 \\
$\mathrm{Ar}$ & & & & & $2.0( \pm 0.8) \times 10^{-6}$ & 6.31 \\
\hline
\end{tabular}

Note. ${ }^{a}$ The second column of abundances for each PN are in logarithmic units, $12+\log _{10}(\mathrm{X} / \mathrm{H})$.

Osterbrock \& Ferland 2006; Liu 2012) and thus the uncertainties of the derived ionic abundances are, to a large extent, subject to the uncertainties in the CEL fluxes. Although the electron density of PN1 may have a relatively large uncertainty, it does not affect the resultant ionic abundances much, as discussed in Section 3.2. For PN2, where the [O III] $\lambda 4363$ line was not observed, a typical nebular electron temperature of $10,000 \mathrm{~K}$ was assumed. Adopting this value may introduce significant uncertainties on the ionic abundances of heavy elements, e.g., $\sim 20 \%-30 \%$ for some ions. Measurement errors on the line fluxes given in Table 2 are mainly estimated from Gaussian profile fits, i.e., the difference between the directly integrated flux and the flux given by the Gaussian fits. Uncertainties of the $\mathrm{He}^{+} / \mathrm{H}^{+}$ionic abundances are dominated by the measurement errors in the line fluxes because the emissivities of the He I recombination lines are much less sensitive to the electron temperature compared to the CELs. The $\mathrm{He}^{+} / \mathrm{H}^{+}$abundances derived from the $\mathrm{He}$ I $\lambda 3888$ line have lower uncertainties than the abundances derived from other He I lines as these other lines have larger measurement uncertainties. However, the $\mathrm{H}_{\mathrm{I}} \lambda 3888$ line is blended with the $\mathrm{H}_{\mathrm{r}} \lambda 3889$ line.

The $\mathrm{O}^{3+}$ ion needs to be taken into account when calculating the $\mathrm{O} / \mathrm{H}$ elemental abundances of our PNe. The determination of $\mathrm{O} / \mathrm{H}$ requires the $\mathrm{He}^{2+} / \mathrm{H}^{+}$ionic abundance (because the ionization potential of $\mathrm{He}^{+}, 54.416 \mathrm{eV}$, is very close to that of the $\mathrm{O}^{2+}$ ion, $54.934 \mathrm{eV}$ ) as derived from the He II $\lambda 4686$ line, which is located at the end of the blue CCD and thus difficult to accurately measure due to the very low $\mathrm{S} / \mathrm{N}$. The upper limits of the $\lambda 4686$ line intensities were estimated, which yields ionization correction factors (ICFs) of oxygen for the three PNe close to unity. The ICF method was developed by Kingsburgh \& Barlow (1994). Since only one ionization stage of nitrogen ([N $\mathrm{II}])$ and neon ([Ne III]) was observed in our $\mathrm{PN}$ sample, the $\mathrm{N} / \mathrm{H}$ elemental abundances were derived from the $\mathrm{O} / \mathrm{H}$ and $\mathrm{O}^{+} / \mathrm{H}^{+}$abundances using Equations (A1) and (A2) in Kingsburgh \& Barlow (1994) and $\mathrm{Ne} / \mathrm{H}$ was derived from $\mathrm{O} / \mathrm{H}$ and $\mathrm{O}^{2+} / \mathrm{H}^{+}$using Equations (A28) and (A29) in Kingsburgh \& Barlow (1994). Only the $\mathrm{S}^{+}$ion was observed in PN3, and the total abundance of sulfur was particularly uncertain. We used Equations (A36)-(A38) in Kingsburgh \& Barlow (1994) to estimate the $\mathrm{S} / \mathrm{H}$ ratio for $\mathrm{PN} 3$. Only the $\mathrm{Ar}^{2+}$ ion was observed in $\mathrm{PN} 3$, and thus the $\mathrm{Ar} / \mathrm{H}$ elemental abundance was estimated using Equations (A32) and (A33) in Kingsburgh \& Barlow (1994). Elemental abundances of N, O, $\mathrm{Ne}, \mathrm{S}$, and Ar are presented in Table 5, with uncertainties given in brackets. Abundance uncertainties were derived directly from the uncertainties in ionic abundances (Table 4), which were estimated based on the measurement uncertainties of the line fluxes and electron temperatures, as discussed earlier in this section. The uncertainties introduced by ionization correction were also taken into account. Regardless of the uncertainties in the ionization correction method for all three PNe, the oxygen abundances are the best calculated of all the heavy elements, while uncertainties in the nitrogen and neon abundances are relatively larger because they were derived from the ionic and total abundances of oxygen. The sulfur and argon abundances are the most uncertain.

\section{DISCUSSION}

Gaseous nebulae, mainly $\mathrm{PNe}$ and $\mathrm{H}$ II regions, are useful probes of the past chemical composition of the interstellar medium (ISM). The $\alpha$-element abundances of a PN reflect those in the ISM at the time when the progenitor star formed, while the $\alpha$-element abundances of a sample of $\mathrm{H}$ II regions provide a "snapshot" of the current status of the chemical evolution of galaxies. A comparison of the abundances of PNe and $\mathrm{H}$ II regions on the disk of a spiral galaxy is instrumental for studying the chemical history and production processes of the elements. Furthermore, studying the relations between abundances of different $\alpha$-elements helps to constrain the production processes and the relative yields of each element. Ratios of different $\alpha$-element abundances also reflect the enrichment by the progenitor stars. Figures 9-15 show the abundance correlations in our sample as well as those for the M31 disk and bulge PNe from the literature, on a logarithmic scale. Figure 9 presents the $\log (\mathrm{N} / \mathrm{O})$ versus $\log (\mathrm{O} / \mathrm{H})$ abundance relation. Also overplotted are the M31 disk PNe from Kwitter et al. (2012) and the M31 bulge and disk sample observed by Jacoby \& Ciardullo (1999). Furthermore, the plot includes the solar values from Asplund et al. (2009) and the nebular abundances of Orion from Esteban et al. (2004). Figure 9 shows that there is no obvious trend in the $\log (\mathrm{N} / \mathrm{O})$ versus $\log (\mathrm{O} / \mathrm{H})$ ratio in our sample.

Figure 10 displays a positive correlation between $\log (\mathrm{Ne} / \mathrm{H})$ and $\log (\mathrm{O} / \mathrm{H})$. This result confirms the tight relation between these two elements, which has been observed for PNe in both the MW and M31. The neon-oxygen abundance distribution of our sample agrees with the slope of other M31 PNe within the errors. Figure 11 shows that there is no obvious correlation between the $\mathrm{Ne} / \mathrm{O}$ ratio and $\mathrm{O} / \mathrm{H}$. Figure 12 shows $\log (\mathrm{S} / \mathrm{H})$ versus $\log (\mathrm{O} / \mathrm{H})$ and Figure 13 shows $\log (\mathrm{S} / \mathrm{O})$ versus $\log (\mathrm{O} / \mathrm{H})$. The sulfur-oxygen correlation shows a larger scatter than the neon-oxygen correlation (Figure 10). As pointed out by Kwitter et al. (2012), as well as by earlier studies, the determination of sulfur abundances in the PNe of M31 is very challenging: (1) ionization corrections introduce uncertainties because of the ions that cannot be observed in the optical, e.g., $\mathrm{S}^{3+}$; and (2) the lines from both $\mathrm{S}^{+}$and the predominant ionization state $\mathrm{S}^{2+}$ are faint, or absent, in the M31 PNe. Our observations (in PN3) confirm the results of Kwitter et al. (2012) that the derived sulfur abundances for the M31 PNe are lower than those of the Sun. The [S II] line is only observed in PN3, and Figures 12 and 13 


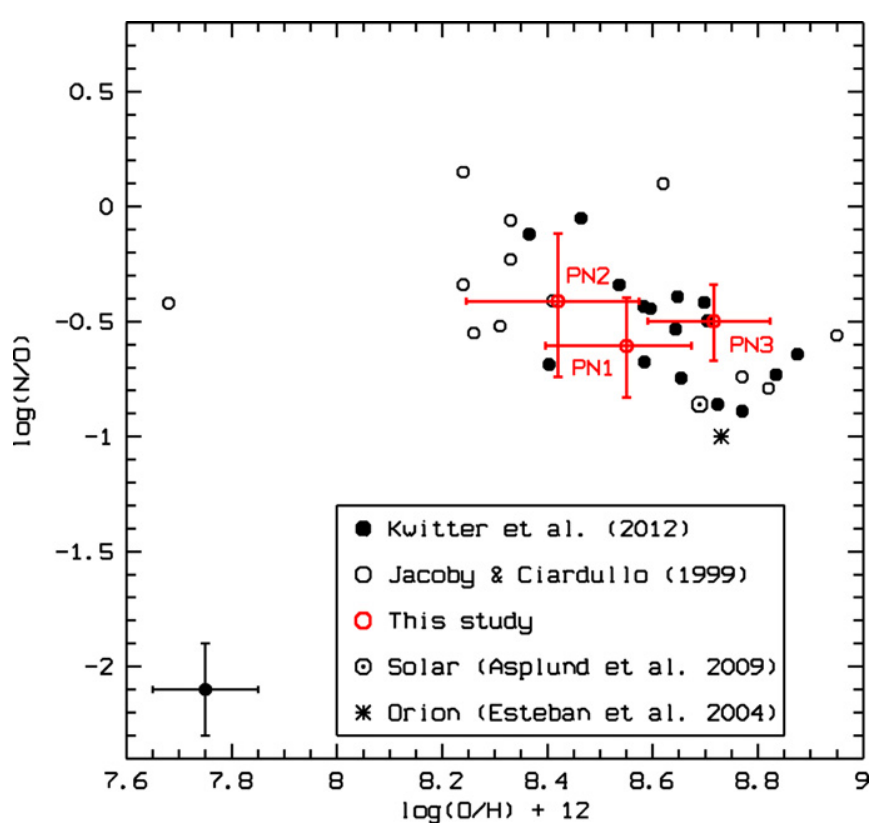

Figure 9. $\mathrm{N} / \mathrm{O}$ vs. $\mathrm{O} / \mathrm{H}$ abundance ratio on a logarithmic scale. Different symbols represent different data sources: the red circles are the three Northern Spur PNe in this study, the black filled circles are the M31 disk PNe from Kwitter et al. (2012), and the black open circles are the M31 bulge and disk PNe from Jacoby \& Ciardullo (1999); the solar value is from Asplund et al. (2009) and the asterisk is the Orion nebular abundance from Esteban et al. (2004). Error bars are given for the three Northern Spur PNe. Representative error bars of the M31 disk PNe observed by Kwitter et al. (2012) are given in the lower left corner.

(A color version of this figure is available in the online journal.)

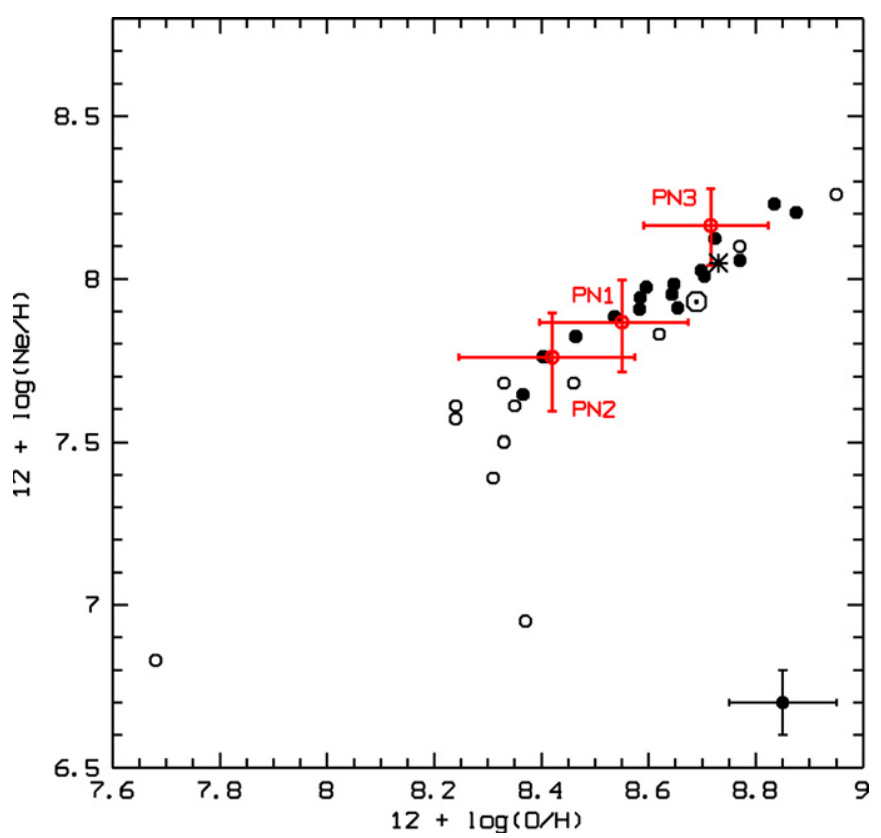

Figure 10. Same as Figure 9 but for $\mathrm{Ne} / \mathrm{H}$ vs. $\mathrm{O} / \mathrm{H}$. Representative error bars of the M31 disk PNe observed by Kwitter et al. (2012) are given in the lower right corner.

(A color version of this figure is available in the online journal.)

show that our abundance ratios lie within the ranges of the Kwitter et al. (2012) results. The behavior of argon is similar to that of neon, as shown in Figure 14, and PN3 is located well within the argon-oxygen correlation of Kwitter et al. (2012). There is no obvious correlation between $\log (\mathrm{Ar} / \mathrm{O})$ and $\log (\mathrm{O} / \mathrm{H})$, as indicated by Figure 15 .

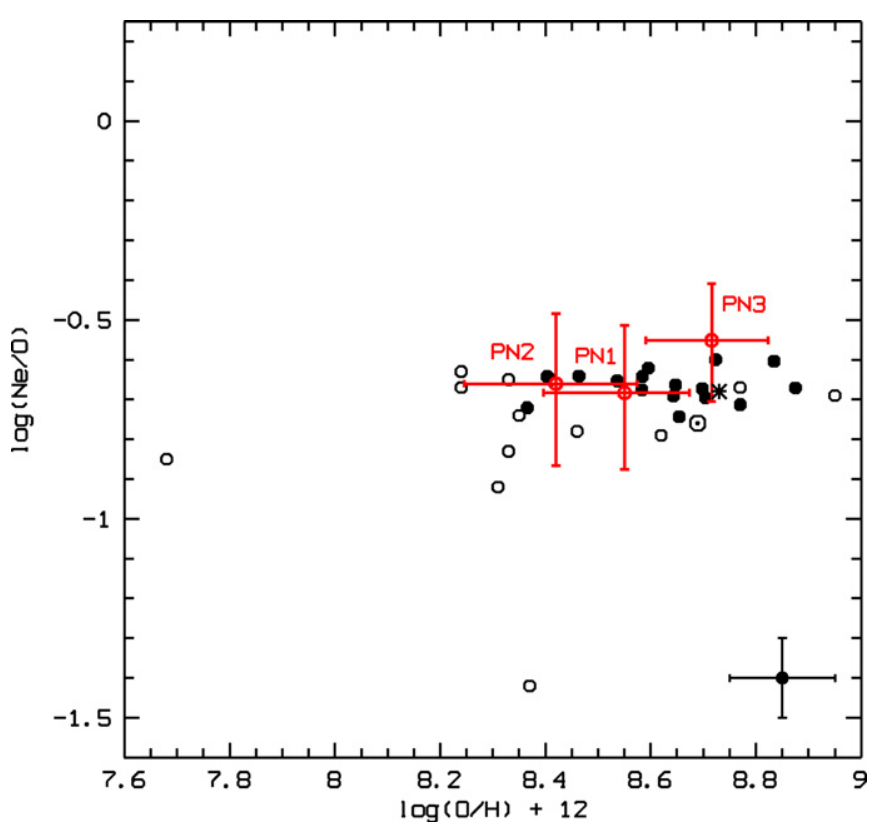

Figure 11. Same as Figure 10 but for $\mathrm{Ne} / \mathrm{O}$ vs. $\mathrm{O} / \mathrm{H}$.

(A color version of this figure is available in the online journal.)

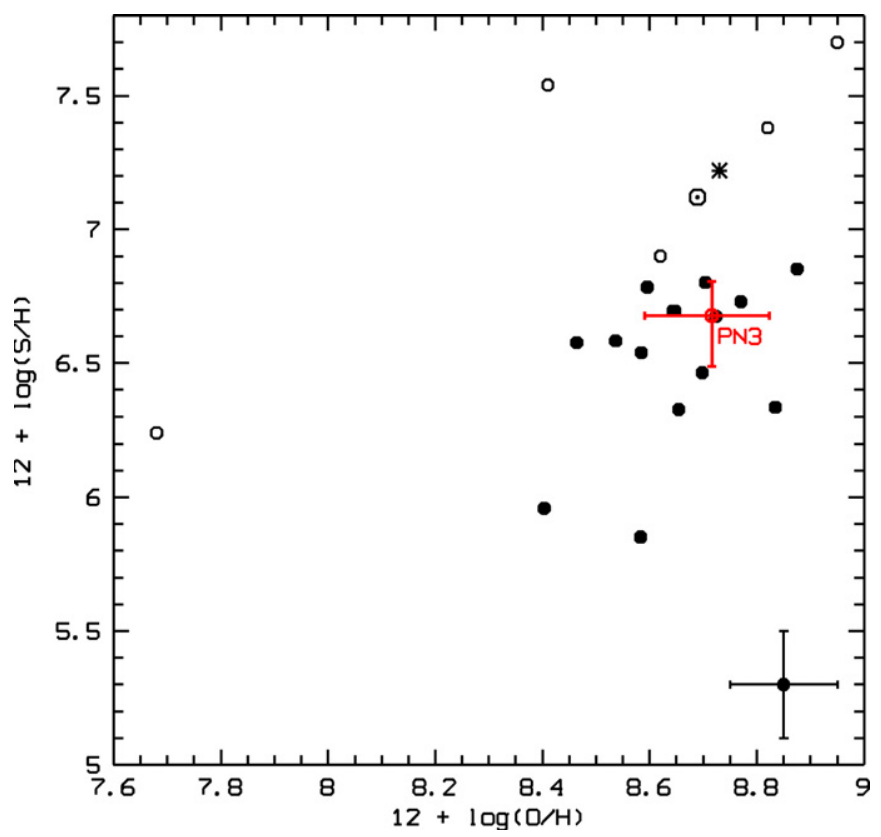

Figure 12. Same as Figure 10 but for $\mathrm{S} / \mathrm{H}$ vs. $\mathrm{O} / \mathrm{H}$. The $\mathrm{S}^{+}$ion is only detected in the spectrum of PN3.

(A color version of this figure is available in the online journal.)

The distribution of oxygen abundance with galactocentric distance for the M31 PNe and $\mathrm{H}$ II regions is shown in Figure 16. The M31 disk sample observed by Kwitter et al. (2012) and our three Northern Spur PNe are both shown in the plot. Also presented are the M31 PNe observed by Sanders et al. (2012) and nine $\mathrm{H}$ II regions on the disk of M31 observed by Zurita \& Bresolin (2012). All galactocentric distances have been rectified for the effects of projection on the sky plane and reduced in units of $R_{25}$, which is $22.4 \mathrm{kpc}$ for M31 (Goodwin et al. 1998). The galactocentric distances (in kpc) of our three PNe have been rectified using the formula

$$
R_{\text {rectified }}=\left(X^{2}+(Y / \cos i)^{2}\right)^{1 / 2}
$$




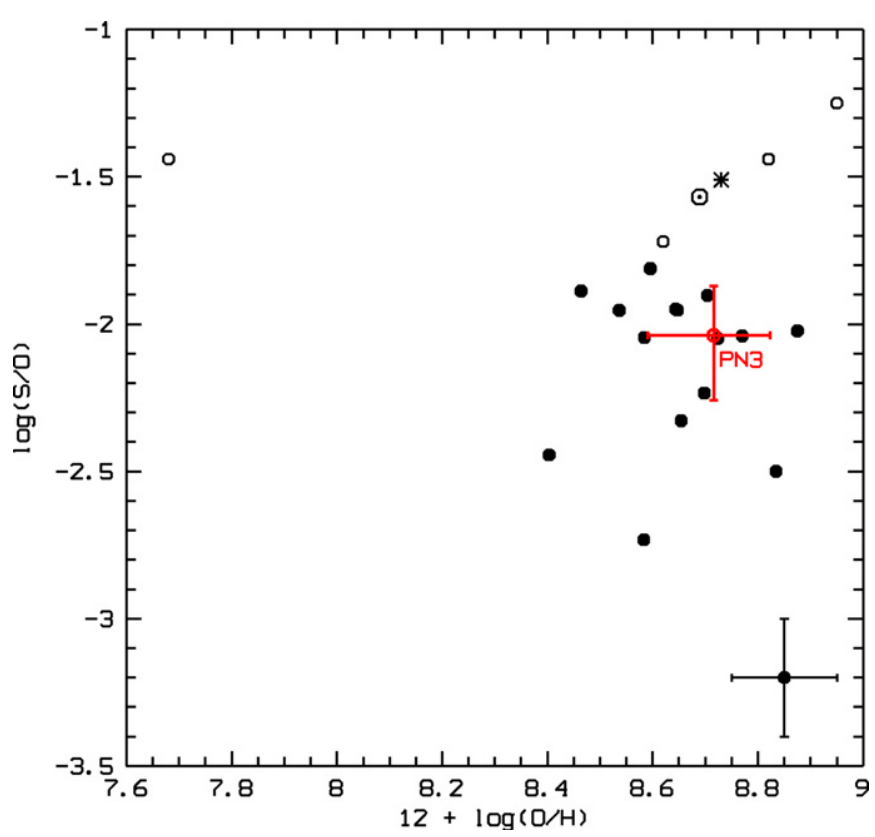

Figure 13. Same as Figure 12 but for $\mathrm{S} / \mathrm{O}$ vs. $\mathrm{O} / \mathrm{H}$.

(A color version of this figure is available in the online journal.)

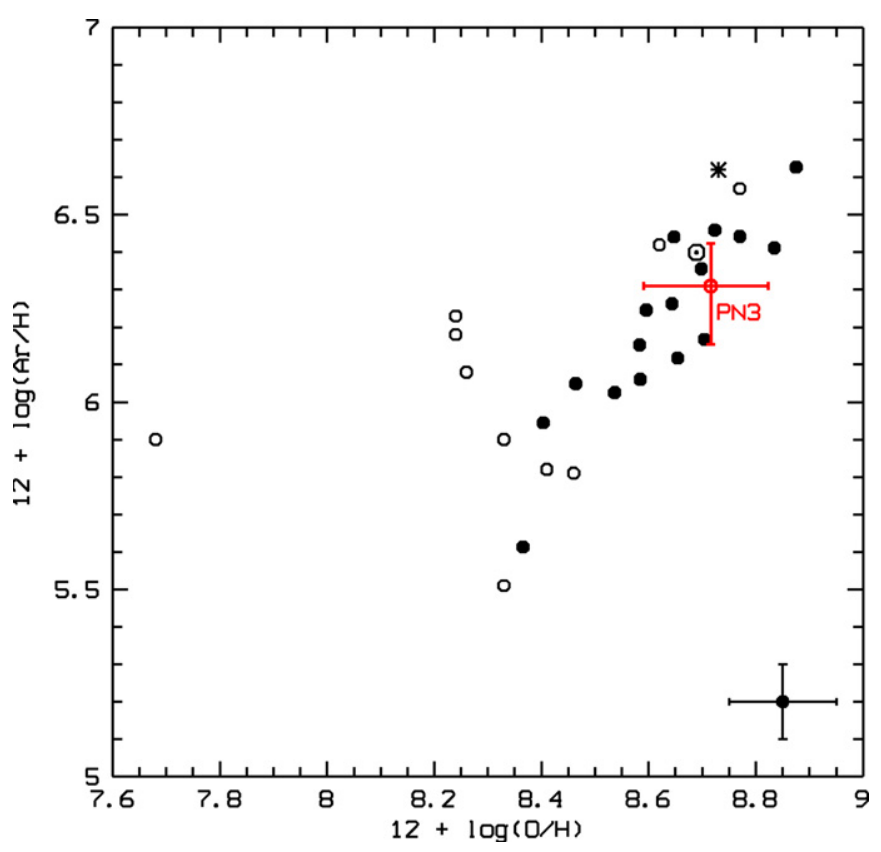

Figure 14. Same as Figure 12 but for $\mathrm{Ar} / \mathrm{H}$ vs. O/H.

(A color version of this figure is available in the online journal.)

given by Kwitter et al. (2012). Here, we assumed that the three Northern Spur PNe are all located on the disk of M31. $X$ and $Y$ are distances to the galactic center projected on the major and minor axes, respectively. The inclination angle $i$ of the M31 disk to the plane of the sky is 77.7 , as adopted from de Vaucouleurs (1958). The $X$ and $Y$ values were calculated from the R.A. and decl. of a PN as well as the position angle of the M31 main axis (37.7; de Vaucouleurs 1958).

We see from Figure 16 that the oxygen abundances of the M31 disk PNe observed by Kwitter et al. (2012) are generally higher than those of Sanders et al. (2012). However, the radial distribution of the sample of Sanders et al. (2012) is more restricted. In our sample, PN3 has a higher oxygen abundance

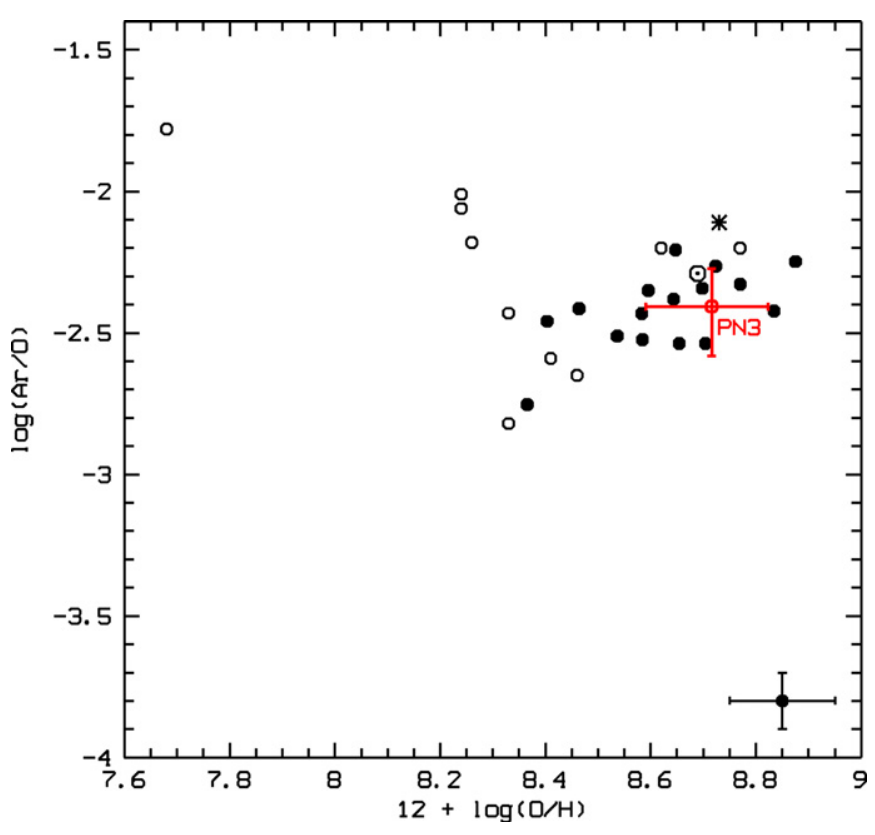

Figure 15. Same as Figure 12 but for $\mathrm{Ar} / \mathrm{O}$ vs. O/H.

(A color version of this figure is available in the online journal.)

than the M31 disk PNe at similar galactocentric distances. This PN also has the best-quality spectrum among the three. This result indicates our sample, at least PN3, might be different from the M31 disk population. Abundances of the sample observed by Sanders et al. (2012) show very large scatter and systematically lower values than those of Kwitter et al. (2012). The nine H II regions in the disk of M31 observed by Zurita \& Bresolin (2012) were derived from the direct $T_{\mathrm{e}}$-based method. These H II regions seem to have steeper oxygen gradients than the objects from Kwitter et al. (2012), but they are spatially restricted and the sample is too small. Judging from the spatial distribution of our three Northern Spur PNe (Figure 17), PN3 may be more associated with this substructure, although all three PNe have already been identified by Merrett et al. (2006) as belonging to the Northern Spur; the other two PNe are located closer to the major axis of M31. If the orbital model of Merrett et al. (2003) is correct, i.e., the Northern Spur of M31 is connected to the Southern Stream, our observations of PN3 seem to be in line with the postulation that the Northern Spur substructure is composed of the tidal debris of M31's satellite galaxies.

The origin of the Northern Spur is still largely unclear, although large-area photometric observations of M31 were carried out more than $10 \mathrm{yr}$ ago. Previous studies of the substructure in M31 have revealed that the Northern Spur is metal-rich. However, those results are only based on color information. Quantitative spectroscopy is needed to confirm that nature. Merrett et al. (2003) were among the first to formally propose the possible origin of the Northern Spur, i.e., it might be associated with the Southern Stream, although that had been inferred by Ferguson et al. (2002) and McConnachie et al. (2003). Using the kinematic information of PNe in the disk of M31 and based on the studies of Ferguson et al. (2002) and McConnachie et al. (2003), Merrett et al. (2003) constructed an orbit model of the stellar stream that connects the Northern Spur to the Southern Stream. In this model, the area of the Northern Spur encompasses the turning point of the orbit that is strongly warped near the center of M31 (see Figure 2 of Merrett et al. 2003; see also Figure 17 in this paper, which was constructed 


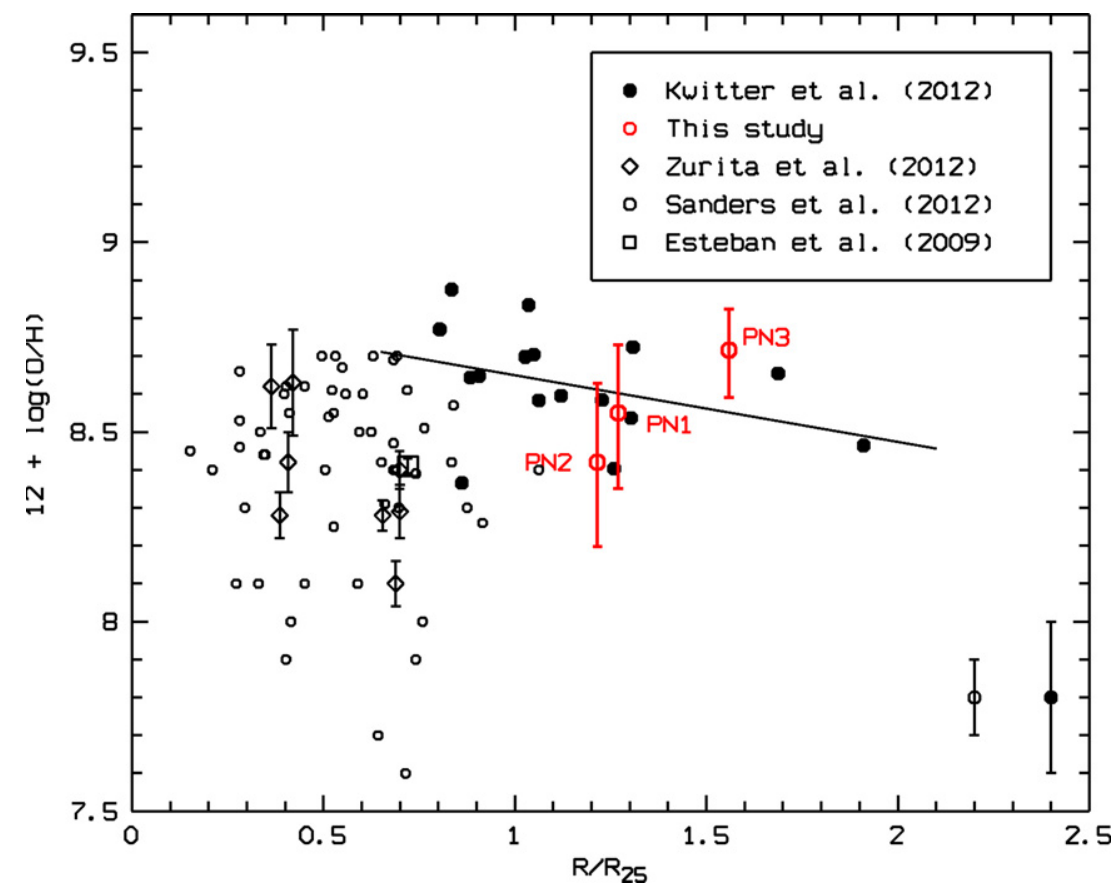

Figure 16. Oxygen gradient of the PNe and $\mathrm{H}$ II regions in M31. The $R_{25}$ value of M31 is $22.4 \mathrm{kpc}$, as adopted from Goodwin et al. (1998). The black filled circles are the M31 disk PNe observed by Kwitter et al. (2012) and the black open circles are the M31 PNe from Sanders et al. (2012). The open diamonds are nine H II regions on the disk of M31 observed by Zurita \& Bresolin (2012) and the claimed abundance uncertainties are given as error bars. The open square is an M31 H II region (K932) observed by Esteban et al. (2009). The three red open circles are our current observations and the abundance uncertainties are given. The galactocentric distances of the three Northern Spur PNe in M31 have been rectified from the sky-projected distances. Representative abundance uncertainties of Kwitter et al. (2012) and Sanders et al. (2012) are indicated in the lower right corner. The black straight line is a simple linear regression fit to the M31 disk PNe (the black filled circles) observed by Kwitter et al. (2012).

(A color version of this figure is available in the online journal.)

based on Figure 2 of Merrett et al. 2003, with permission from the authors). Judging from the projected position and kinematics of M32, Merrett et al. (2003) hypothesized that this satellite might be a parent of the stream, although the exact position of M32 with respect to M31 is still an open question.

Figure 17 shows this orbit in the $X-Y$ coordinate system in an M31-based reference frame, where $X$ lies along the major axis of M31 and increases toward the southwest, and $Y$ lies along the minor axis and increases toward the northwest. Both coordinates were calculated following the geometric transformations of Huchra et al. (1991). Also presented in Figure 17 is the projection of this orbit in the line-of-sight velocity with respect to M31, $v_{\text {los }}$, versus distance along the major and minor axes. Spatial and kinematic distributions of the M31 PNe observed by Merrett et al. (2006) are presented along with the orbit. PNe in the region of the Northern Spur identified by Merrett et al. (2006; see Figure 32 therein), as well as those identified by the same authors as forming a continuation of the Southern Stream, are highlighted with different symbols in Figure 17. The three Northern Spur PNe studied in the current paper are also highlighted. In the lower panel of Figure 17 (i.e., $v_{\text {los }}$ versus $X$ ), the dispersion in the line-of-sight velocities of the Northern Spur PNe with respect to the projected orbit is relatively large, indicating the orbit in this section might have a large uncertainty. This result is expected because the orbit model of Merrett et al. (2003) is based on a limited number of PNe ( 20) and relatively simple assumptions. Considering the fact that PN3 in our sample has relatively higher oxygen abundance than the M31 disk sample at similar galactocentric distances (Figure 16), our observations seem to be in line with the postulation of Merrett et al. (2003). Also noticeable in Figure 17 (also Figure 32 in Merrett et al. 2006) is that the $20 \mathrm{PNe}$ (two of them might be
Northern Spur candidates, as indicated by Merrett et al. 2006) associated with the Southern Stream generally fit the orbit model well. Future spectroscopy of these PNe will help to confirm their true nature. The kinematics of PNe in the Northern Spur region are indistinguishable from those of the disk, and Merrett et al. (2006) suggest that this substructure is related to the disk, perhaps indicative of a warp. However, deep spectroscopy of more PNe in this region is definitely needed so that the PNe can be chemically distinguished from those in the disk. Figure 17 visually shows the position of the Northern Spur PNe relative to the model orbit of Merrett et al. (2003). As to the origin of the Northern Spur, observations of more PNe therein and the PNe in the Southern Stream may help to confirm whether these two substructures are linked. Observations of the PNe in M32 may also help to elucidate the interaction between M31 and M32 and assess whether the Northern Spur and Southern Stream both originate from M32. Currently, observations of PNe in M32 are scarce (Richer et al. 1999) and results are inconclusive. More high-quality spectroscopic observations are preferred.

\section{SUMMARY AND CONCLUSIONS}

We present deep spectroscopy of three PNe in the Northern Spur of M31 using the DBSP on the $5.1 \mathrm{~m}$ Hale Telescope at the Palomar Observatory. The sample is selected from Merrett et al. (2006). This study is the first chemical investigation of $\mathrm{PNe}$ in this substructure. The [O III] $\lambda 4363$ auroral line is detected in the spectra of two objects after meticulous work on the subtraction of the sky background. Electron temperatures were determined for two PNe. Ionic abundances of heavy elements were derived from the [N II], [O III], [Ne III], [S II], and [Ar III] CELs detected in the spectra. We estimate the $\mathrm{N} / \mathrm{H}, \mathrm{O} / \mathrm{H}, \mathrm{Ne} / \mathrm{H}$, 


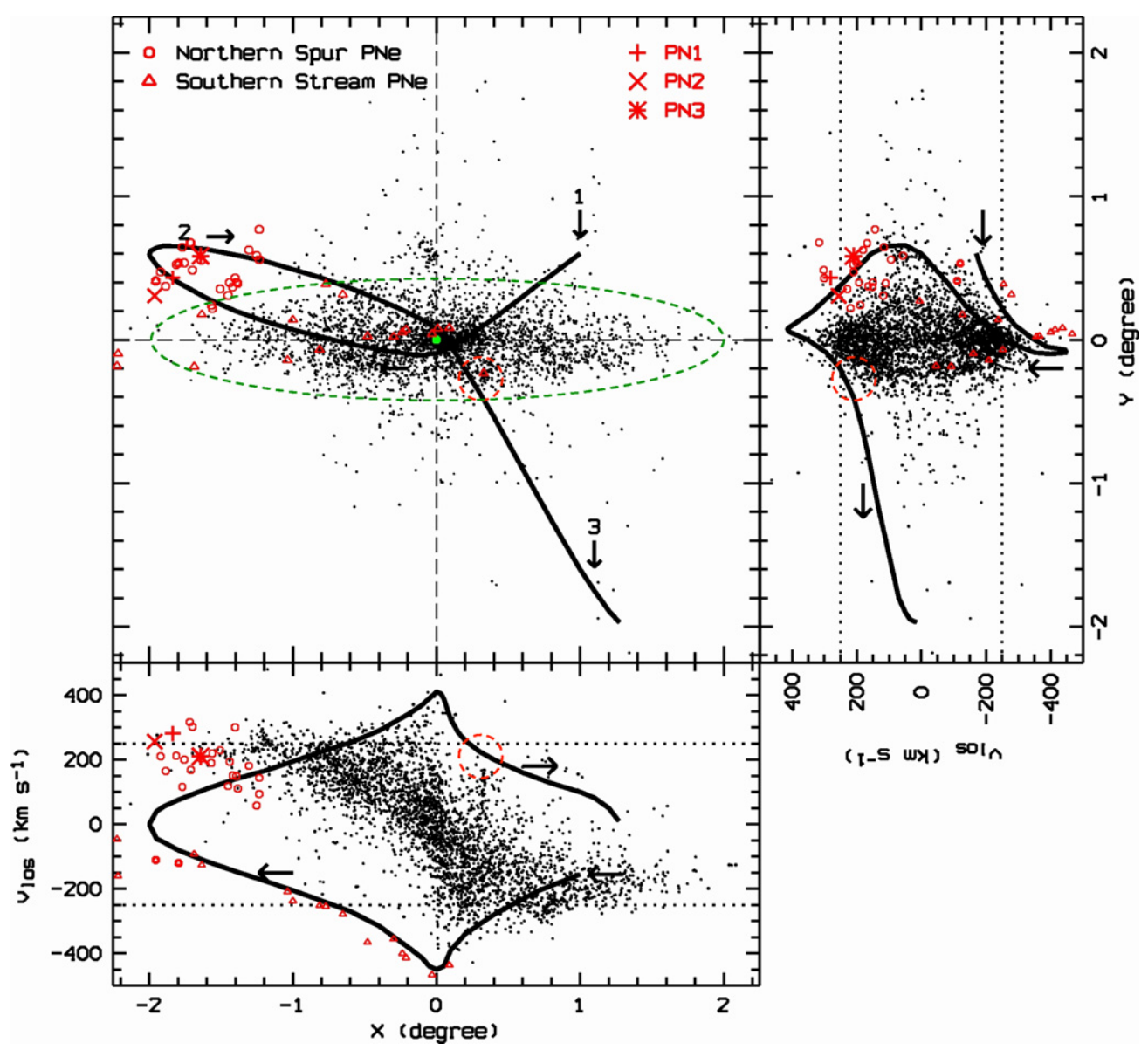

Figure 17. Spatial distribution and kinematics of PNe in M31 (see the description of the $X$ and $Y$ coordinates in the text). Samples are from Merrett et al. (2006). Small red circles represent the Northern Spur PNe and red triangles are those identified by Merrett et al. (2006) as forming a continuation of the Southern Stream. The three Northern Spur PNe studied in the current paper are highlighted. In the upper left panel: overplotted is the orbit (thick black curve) proposed by Merrett et al. (2003); the green dot represents the center of M31 and the green dashed ellipse has a semimajor axis of $2^{\circ}(\sim 27 \mathrm{kpc})$ and represents a disk with an inclination angle $i=77.7$ (de Vaucouleurs 1958). In the two side panels (lower and right): a projection of the orbit in line-of-sight velocity with respect to M31, $v_{\text {los }}$, vs. distance along the major and minor axes of M31 is superimposed on the PNe data. The arrows show the direction along the stream. The red dashed circle shows the location of M32. The format of the figure follows Figure 2 in Merrett et al. (2003), and the orbit is reproduced based on that figure (with permission from the authors).

(A color version of this figure is available in the online journal.)

$\mathrm{S} / \mathrm{H}$, and $\mathrm{Ar} / \mathrm{H}$ elemental abundances. Correlations between oxygen and $\alpha$-element abundance ratios were studied, using our sample and other M31 PNe from the literature. One of the three Northern Spur PNe has a relatively higher oxygen abundance than both the M31 disk PNe at similar galactocentric distances and the average abundance of the M31 disk sample. Our study thus seems to be in line with the postulation that the Northern Spur might be connected to the Southern Stream and is metal enriched. More observations of PNe at different substructures, in combination with kinematic information, are needed to assess the properties of those substructures and to further constrain the possible origins of the Northern Spur.

This research uses data obtained through the Telescope Access Program (TAP), which is funded by the National Astronomical Observatories, Chinese Academy of Sciences, and the Special Fund for Astronomy from the Ministry of Finance. Observations carried out with the $5.1 \mathrm{~m}$ Hale Telescope at the Palomar Observatory were obtained as part of an agreement between the National Astronomical Observatories, Chinese Academy of Sciences, and the California Institute of Technology. The project is also supported by the National Science Foundation of China (No. 10933001). X.F. and Y.Z. thank the staff of the
Palomar Observatory for kind help during observations. Y.Z. thanks the Research Grants Council of the Hong Kong Special Administrative Region, China for financial support (Grants HKU7073/11P). R.G.B. acknowledges support from MICINN AYA2010-1508 and China National Postdoc Fund (Grant No. 20100480144). We thank Martin A. Guerrero and Enrique Pérez from the Instituto de Astrofísica de Andalucía (IAA-CSIC) for valuable comments and suggestions. We also thank Michael R. Merrifield from the University of Nottingham for giving us permission to produce a figure (Figure 17 in this paper) based on Figure 2 in Merrett et al. (2003). We also thank the anonymous referee whose comments have greatly improved the quality of this article.

\section{REFERENCES}

Abadi, M. G., Navarro, J. F., \& Steinmetz, M. 2006, MNRAS, 365, 747 Asplund, M., Grevesse, N., Sauval, A.-J., \& Scott, P. 2009, ARA\&A, 47, 481 Benjamin, R. A., Skillman, E. D., \& Smits, D. P. 1999, ApJ, 514, 307 Benjamin, R. A., Skillman, E. D., \& Smits, D. P. 2002, ApJ, 569, 288 Cardelli, J. A., Clayton, G. C., \& Mathis, J. S. 1989, ApJ, 345, 245 de Vaucouleurs, G. 1958, ApJ, 128, 465 de Vaucouleurs, G., de Vaucouleurs, A., Corwin, H. G., Jr., et al. 1991, Third Reference Catalogue of Bright Galaxies (New York: Springer) Esteban, C., Bresolin, F., Peimbert, M., et al. 2009, ApJ, 700, 654 
Esteban, C., Peimbert, M., García-Rojas, J., et al. 2004, MNRAS, 355, 229 Ferguson, A. M. N., Irwin, M. J., Ibata, I. A., Lewis, G. F., \& Tanvir, N. R. 2002, AJ, 124, 1452

Ford, H. C., \& Jacoby, G. H. 1978a, ApJ, 219, 437

Ford, H. C., \& Jacoby, G. H. 1978b, ApJS, 38, 351

Goodwin, S. P., Gribbin, J., \& Henry, M. A. 1998, Obs, 118, 201

Huchra, J. P., Brodie, J. P., \& Kent, S. M. 1991, ApJ, 370, 495

Ibata, R. A., Irwin, M. J., Lewis, G. F., Ferguson, A. M. N., \& Tanvir, N. R. 2001a, Natur, 412, 49

Ibata, R. A., Irwin, M. J., Lewis, G. F., \& Stotle, A. 2001b, ApJL, 547, L133

Ibata, R. A., Martin, N. F., Irwin, M. J., et al. 2007, ApJ, 671, 1591

Innanen, K. A., Kamper, K. W., van den Bergh, S., \& Papp, K. A. 1982, ApJ, 254,515

Irwin, M. J., Ferguson, A. M. N., Ibata, R. A., Lewis, G. F., \& Tanvir, N. R. 2005, ApJL, 628, L105

Jacoby, G. H., \& Ciardullo, R. 1999, ApJ, 515, 169

Jacoby, G. H., \& Ford, H. C. 1986, ApJ, 304, 490

Kingsburgh, R. L., \& Barlow, M. J. 1994, MNRAS, 271, 257

Kwitter, K. B., Lehman, E. M. M., Balick, B., \& Henry, R. B. C. 2012, ApJ, 753,12

Liu, X.-W. 2012, in New Vision 400: Engaging Big Questions in Astronomy and Cosmology Four Hundred Years after the Invention of the Telescope, ed. D. G. York, O. Gingerich, \& S.-N. Zhang (Boca Raton, FL: CRC Press), 103

Majewski, S. R., Skrutskie, M. F., Weinberg, M. D., \& Ostheimer, J. C. 2003, ApJ, 599, 1082

McConnachie, A. W., Irwin, M. J., Ferguson, A. M. N., et al. 2005, MNRAS, 356,979
McConnachie, A. W., Irwin, M. J., Ibata, R. A., et al. 2003, MNRAS, 343, 1335 McConnachie, A. W., Irwin, M. J., Ibata, R. A., et al. 2009, Natur, 461, 66

McConnachie, A. W., Irwin, M. J., Lewis, G. F., et al. 2004, MNRAS, 351, L94

Merrett, H. R., Kuijken, K., Merrifield, M. R., et al. 2003, MNRAS, 346, L62

Merrett, H. R., Merrifield, M. R., Douglas, N. G., et al. 2006, MNRAS, 369,120

Newton, K., \& Emerson, D. T. 1977, MNRAS, 181, 573

Oke, J. B. 1990, AJ, 99, 1621

Osterbrock, D. E., \& Ferland, G. J. 2006, Astrophysics of Gaseous Nebulae and Active Galactic Nuclei (Sausalito, CA: Univ. Science Books)

Pérez, E., Cid Fernandez, R., González Delgado, R. M., et al. 2013, ApJL, 764, L1

Richer, M. G., Stasińska, G., \& McCall, M. L. 1999, A\&AS, 135, 203

Robbins, R. R. 1968, ApJ, 151, 511

Sanders, N. E., Caldwell, N., McDowell, J., \& Harding, P. 2012, ApJ, 758,133

Sansonetti, C. J., Salit, M. L., \& Reader, J. 1996, ApOpt, 35, 74

Schlegel, D. J., Finkbeiner, D. P., \& Davis, M. 1998, ApJ, 500, 525

Stasińska, G., Richer, M. G., \& McCall, M. L. 1998, A\&A, 336, 667

Storey, P. J., \& Hummer, D. G. 1995, MNRAS, 272, 41

Walterbos, R. A. M., \& Kennicutt, R. C., Jr. 1988, A\&A, 198, 61

White, S. D. M. 1978, MNRAS, 184, 185

White, S. D. M., \& Rees, M. J. 1978, MNRAS, 183, 341

Yuan, H.-B., Liu, X.-W., Huo, Z.-Y., et al. 2010, RAA, 10, 599

Zhang, Y., Liu, X.-W., \& Rubin, R. H. 2005, MNRAS, 358, 457

Zurita, A., \& Bresolin, F. 2012, MNRAS, 427, 1463 